\title{
Atlas of marine bony fish otoliths (sagittae) of Southeastern-Southern Brazil Part VII: Atheriniformes, Beloniformes, Beryciformes, Zeiformes, Syngnathiformes, Scorpaeniformes and Tetraodontiformes
}

\author{
Valéria Regina Martins Conversani ${ }^{1}$, Marina Rito Brenha-Nunes ${ }^{1}$, César Santificetur ${ }^{l}$, Marcella \\ Bockis Giaretta ${ }^{l}$, Carolina Correia Siliprandi ${ }^{l}$, Carmen Lucia Del Bianco Rossi-Wongtschowski ${ }^{{ }^{*}}$ \\ ${ }^{1}$ Instituto Oceanográfico da Universidade de São Paulo \\ (Praça do Oceanográfico, 191, 05508-120 São Paulo, SP, Brazil) \\ *Corresponding author: cwongski@usp.br
}

\section{Abstract}

In addition to the series of documents that we have been publishing on the "Atlas of Teleostei Otoliths for the Southeastern-Southern Brazilian region", in this volume we present the results of species of the orders Atheriniformes (1 species), Beloniformes (5), Beryciformes (2), Zeiformes (2), Syngnathiformes (2), Scorpaeniformes (9) and Tetraodontiformes (6). Features, measurements and indices were analyzed according to methodology used in anterior series. Three otoliths of each species have been illustrated and photographed whenever possible. The frequency of occurrence of each characteristic was calculated by total length classes (TL), and differences within and among classes have been analyzed applying the multiple $\chi^{2}$ test (significance 0.05 ).

Descriptors: Otoliths, Morphology, Morphometry, Southwestern Atlantic, Brazil.

\section{Resumo}

Em adição à série de documentos que estamos publicando sobre o "Atlas de Otólitos para os peixes Teleósteos da região Sudeste-Sul do Brasil", neste volume apresentamos os resultados obtidos para espécies das ordens Atheriniformes (1 espécie), Beloniformes (5), Beryciformes (2), Zeiformes (2), Syngnathiformes (2), Scorpaeniformes (9) e Tetraodontiformes (6). Foram analisadas as feições, medidas e índices usualmente empregados conforme metodologia apresentada nas séries anteriores. Três otólitos de cada espécie foram desenhados e fotografados, sempre que possível. A frequência de ocorrência de cada característica por classe de comprimento total foi calculada (CT) e as diferenças dentro de cada classe e entre classes foram analisadas aplicando teste $\chi^{2}$ múltiplo (significância 0,05 ).

Descritores: Otólitos, Morfologia, Morfometria, Atlântico Sudoeste, Brasil. 


\section{INTRODUCTION}

Otolith shape analyses are helpful indicators for community, population and individual analysis and environmental events.

Since 2014, as a contribution for these and other studies we have been improving the Collection of Teleostei Fish Otoliths of the Southeastern/Southern Brazilian region (COSS Brasil) held at the Instituto Oceanográfico USP, (IOUSP). Currently, the collection contains 51,886 pairs of otoliths from 66 families, 24 orders and 202 species (ROSSI-WONGTSCHOWSKI et al., 2016). Until now, we published results on the shape of other orders and many families in ROSSI-WONGTSCHOWSKI et al. (2014), SILIPRANDI et al. (2016) and BRENHA-NUNES et al. (2016). These publications will constitute an Atlas of Otoliths of the Southeastern/Southern Brazilian region.

In this volume, we present results of the morphology and morphometry of the otoliths of species belonging to the orders Atheriniformes, Beloniformes, Beryciformes, Zeiformes, Syngnathiformes, Scorpaeniformes and Tetraodontiformes. We hope that our papers and our collection will contribute to all kinds of studies involving otolith morphology.

\section{MATERIAL AND METHODS}

The sampled area and the methodology of this study followed that presented in ROSSI-WONGTSCHOWSKI et al. (2014), SILIPRANDI et al. (2016) and BRENHA-NUNES et al. (2016).

The acronyms in the shape indices tables represent: $\mathrm{TL}=$ total fish length, $\mathrm{OL}=$ otolith length, $\mathrm{OH}=$ otolith height and OT=otolith thickness.

\section{RESULTS}

\section{ORDER ATHERINIFORMES}

\section{FAMILY ATHERINOPSIDAE}

Atherinella brasiliensis (Quoy \& Gaimard 1825) - Plate 1

\begin{tabular}{ll}
\hline $\begin{array}{l}\text { Maximum Size: } \\
\text { Distribution: }\end{array}$ & $160 \mathrm{~mm}$ (TL) (FROSE; PAULY, 2016). \\
Habitat: & Western Atlantic, along the South America coast (CARPENTER; MUNROE, 2015). \\
Diet: & Benthopelagic; occurs in estuaries, mangroves and soft bottoms (CHERNOFF, 2002). \\
Collection: & Feeds mainly on zooplankton and diatoms (CONTENTE et al., 2011). \\
Sample: & 1014 otoliths from 541 fishes (TL ranging from 17 to $151 \mathrm{~mm}$ ). \\
\hline
\end{tabular}

Shape: elliptic. Anterior region: peaked (45\%), peaked-round, notched, angled-round. Posterior region: round (76\%), angled, angled-round. Dorsal edge: lobed (47\%), sinuate, entire, lobed to sinuate. Ventral edge: lobed (44\%), sinuate (38\%), lobed to entire, entire. Profile: concave-convex. Rostrum and antirostrum orientation: in agreement (85\%), does not apply. Rostrum: developed (78\%), underdeveloped. Antirostrum: underdeveloped (60\%), developed, absent. Pseudorostrum: absent. Pseudoantirostrum: absent. Sulcus acusticus: position: supramedian (73\%), median; orientation: ascending (76\%), horizontal; opening: ostial; morphology: heterosulcoid; colliculum: heteromorphic; ostium: elliptic $(62 \%)$, funnel-like; cauda: tubular sinuous. 
Statistical differences $(p<0.05)$ within some length classes were obtained for dorsal and ventral edges, anterior and posterior regions, rostrum and antirostrum development and orientation, sulcus acusticus orientation and position and ostium morphology. Along the fish growth statistical differences were found for dorsal and ventral edges, anterior and posterior regions, sulcus acusticus position and orientation, ostium morphology and rostrum development.

\begin{tabular}{lccc}
\hline Shape indices & Mean \pm Sd & Minimum & Maximum \\
\hline OL/TL (\%) & $2.99 \pm 0.27$ & 2.64 & 4.21 \\
OH/OL (\%) & $60.52 \pm 2.85$ & 53.52 & 68.54 \\
OT/OL (\%) & $19.41 \pm 2.28$ & 16.38 & 29.21 \\
OT/OH (\%) & $32.06 \pm 3.18$ & 25.82 & 43.55 \\
Circularity & $16.76 \pm 1.41$ & 14.82 & 20.72 \\
Rectangularity & $0.73 \pm 0.02$ & 0.69 & 0.77 \\
\hline
\end{tabular}

\section{ORDER BELONIFORMES}

\section{FAMILY HEMIRAMPHIDAE}

The anterior region tends to be peaked, the sulcus acusticus orientation is ascending, the ostium is frequently elliptic and the cauda morphology is tubular slightly curved in most of otoliths. The pseudorostrum and pseudoantirostrum are always absent.

Hemiramphus brasiliensis (Linnaeus 1758) - Plate 2

\begin{tabular}{ll}
$\begin{array}{l}\text { Maximum Size: } \\
\text { Distribution: }\end{array}$ & $\begin{array}{l}550 \mathrm{~mm} \text { (TL) (SCHNEIDER, 1990). } \\
\text { Western Atlantic, from United States to Brazil and Eastern Atlantic from Canary Islands to Luanda } \\
\text { (COLLETTE, 2002a). }\end{array}$ \\
Habitat: & Shallow coastal waters, commonly forming schools (FIGUEIREDO; MENEZES, 1978). \\
Diet: & Feeds on sea grasses and small fishes (FIGUEIREDO; MENEZES, 1978). \\
Collection: & 34 otoliths from 17 fishes (TL ranging from 116 to $200 \mathrm{~mm})$. \\
Sample: & 13 left otoliths categorized into $6,20 \mathrm{~mm}$ classes $(100$ to $220 \mathrm{~mm})$. \\
\hline
\end{tabular}

Shape: elliptic to oval. Anterior region: peaked-round (92\%), angled-round. Posterior region: oblique-round (54\%), round, angled. Dorsal edge: lobed to sinuate (92\%), dentate to lobed. Ventral edge: lobed (62\%), lobed to sinuate, dentate to lobed. Profile: concave-convex. Rostrum and antirostrum orientation: does not apply. Rostrum: developed (85\%), underdeveloped, absent. Antirostrum: absent. Sulcus acusticus: position: median; orientation: ascending; opening: pseudo-ostial; morphology: heterosulcoid; colliculum: heteromorphic; ostium: elliptic (62\%), tubular; cauda: tubular slightly curved.

Statistical differences $(p<0.05)$ within some length classes were obtained for dorsal edge, anterior region and rostrum development. No differences were found along the fish development. 


\begin{tabular}{lccc}
\hline Shape indices & Mean \pm Sd & Minimum & Maximum \\
\hline OL/TL (\%) & $2.20 \pm 0.14$ & 1.92 & 2.51 \\
OH/OL (\%) & $70.44 \pm 3.03$ & 66.42 & 76.10 \\
OT/OL (\%) & $16.68 \pm 1.09$ & 15.38 & 19.18 \\
OT/OH (\%) & $23.69 \pm 1.35$ & 21.53 & 25.93 \\
Circularity & $16.14 \pm 4.64$ & 0.97 & 18.77 \\
Rectangularity & $0.72 \pm 0.02$ & 0.69 & 0.76 \\
\hline
\end{tabular}

Hyporhamphus roberti (Valenciennes 1847) - Plate 3

\begin{tabular}{ll}
\hline $\begin{array}{l}\text { Maximum Size: } \\
\text { Distribution: }\end{array}$ & $320 \mathrm{~mm}$ (TL) (CERVIGÓN et al., 1992). \\
& $\begin{array}{l}\text { Central and South America coast, from Panama to Southeastern Brazil (FIGUEIREDO; MENEZES, 1978; } \\
\text { COLLETTE, 2002a). }\end{array}$ \\
Habitat: & Estuaries and river mouths (COLLETTE, 2002a). \\
Diet: & Feeds on seagrass, small crustaceans and mollusks (FIGUEIREDO; MENEZES, 1978). \\
Collection: & 2 otoliths from $1 \mathrm{fish}(\mathrm{TL}: 132 \mathrm{~mm})$. \\
Sample: & 1 left otolith $(132 \mathrm{~mm})$. \\
\hline
\end{tabular}

Shape: elliptic. Anterior region: peaked. Posterior region: angled. Dorsal edge: sinuate. Ventral edge: entire. Profile: concave-convex. Rostrum and antirostrum orientation: does not apply. Rostrum: underdeveloped. Antirostrum: absent. Sulcus acusticus: position: median; orientation: ascending; opening: para-ostial; morphology: heterosulcoid; colliculum: heteromorphic; ostium: elliptic; cauda: tubular slightly curved.

Only one otolith was examined not allowing statistical analysis of the data but its morphometric characteristics are shown below:

\begin{tabular}{lccc}
\hline Shape indices & Mean \pm Sd & Minimum & Maximum \\
\hline OL/TL (\%) & $2.01 \pm 0$ & 2.01 & 2.01 \\
OH/OL (\%) & $63.77 \pm 0$ & 63.77 & 63.77 \\
OT/OL (\%) & $16.23 \pm 0$ & 16.23 & 16.23 \\
OT/OH (\%) & $25.44 \pm 0$ & 25.44 & 25.44 \\
Circularity & $14.97 \pm 0$ & 14.97 & 14.97 \\
Rectangularity & $0.71 \pm 0$ & 0.71 & 0.71 \\
\hline
\end{tabular}

Hyporhamphus unifasciatus (Ranzani 1841) - Plate 4

\begin{tabular}{ll}
\hline Maximum Size: & $300 \mathrm{~mm}$ (TL) (CERVIGÓN et al., 1992). \\
Distribution: & Western Atlantic, from South Florida to Uruguay (COLLETTE, 2002a). \\
Habitat: & Inshore surface, forming schools (COLLETTE, 2002a). \\
Diet: & Feeds on seagrass, small crustaceans and mollusks (FIGUEIREDO; MENEZES, 1978). \\
Collection: & 102 otoliths from 51 fishes (TL ranging from 116 to $282 \mathrm{~mm}$ ). \\
Sample: & 33 left otoliths categorized into 7, $20 \mathrm{~mm}$ classes (100 to $280 \mathrm{~mm}$ ). \\
\hline
\end{tabular}

Shape: oval to elliptic (52\%), elliptic (48\%). Anterior region: peaked-round. Posterior region: oblique to angled (73\%), oblique-round, oblique, round. Dorsal edge: lobed to sinuate (70\%), crenate to entire, sinuate to entire, lobed. Ventral edge: lobed to sinuate (42\%), sinuate to entire, lobed, crenate to entire. Profile: concave-convex. Rostrum and antirostrum orientation: does not apply (64\%), in agreement. Rostrum: developed. Antirostrum: absent (64\%), underdeveloped. Sulcus acusticus: position: median; orientation: ascending; opening: para-ostial (64\%), pseudo-ostial; morphology: heterosulcoid; colliculum: heteromorphic; ostium: elliptic; cauda: tubular slightly curved (85\%), tubular strongly curved. 
Statistical differences $(p<0.05)$ within some length classes were obtained for dorsal edge, posterior region and cauda morphology. No differences were found along the fish development.

\begin{tabular}{lccc}
\hline Shape indices & Mean \pm Sd & Minimum & Maximum \\
\hline OL/TL (\%) & $2.28 \pm 0.17$ & 2.05 & 2.93 \\
OH/OL (\%) & $67.86 \pm 3.76$ & 59.02 & 74.86 \\
OT/OL (\%) & $16.22 \pm 0.98$ & 14.47 & 18.40 \\
OT/OH (\%) & $23.95 \pm 1.59$ & 20.66 & 28.42 \\
Circularity & $17.45 \pm 1.24$ & 15.16 & 22.51 \\
Rectangularity & $0.71 \pm 0.02$ & 0.67 & 0.75 \\
\hline
\end{tabular}

\section{FAMILY BELONIDAE}

The otolith shape is frequently rectangular, although Strongylura timucu tends to be elliptic to rectangular. The rostrum is always developed and the ostium is frequently funnel-like. The pseudorostrum and pseudoantirostrum are always absent.

\section{Strongylura marina (Walbaum 1792) - Plate 5}

\begin{tabular}{ll}
\hline $\begin{array}{l}\text { Maximum Size: } \\
\text { Distribution: }\end{array}$ & $111 \mathrm{~mm}$ (TL) (IGFA, 2001), but attaining $621 \mathrm{~mm}$ in our collection. \\
Habitat: & Wester Atlantic, from Maine, USA to Southern Brazil (FIGUEIREDO; MENEZES, 1978). \\
& Coastal areas, mangrove-lined lagoons and also enters fresh water (FIGUEIREDO; MENEZES, 1978; \\
COLLETTE, 2002b). & \\
Diet: & Feeds on small fishes and crustaceans (COLLETTE, 2002b). \\
Collection: & 13 otoliths from 7 fishes (TL ranging from 180 to $621 \mathrm{~mm})$. \\
Sample: & 5 left otoliths categorized into 4, $20 \mathrm{~mm}$ classes (280 to $640 \mathrm{~mm})$. \\
\hline
\end{tabular}

Shape: rectangular ( $80 \%$ - small fish), trapezoidal (large fish). Anterior region: double-peaked (80\%), peaked. Posterior region: oblique-round (40\%), flattened (40\%), round. Dorsal edge: crenate to lobed (40\%), lobed to sinuate (40\%), crenate to entire. Ventral edge: lobed (60\%), dentate to lobed. Profile: plane-convex (80\%), concave-convex. Rostrum and antirostrum orientation: in agreement (80\%), does not apply. Rostrum: developed. Antirostrum: developed (80\%), absent. Sulcus acusticus: position: median (80\%), supramedian; orientation: ascending; opening: ostial; morphology: heterosulcoid; colliculum: heteromorphic; ostium: funnel-like (80\%), elliptic; cauda: tubular straight.

The small number of otoliths examined did not permit the statistical analysis of the data but its morphometric characteristics are shown below:

\begin{tabular}{lccc}
\hline Shape indices & Mean \pm Sd & Minimum & Maximum \\
\hline OL/TL (\%) & $0.91 \pm 0.02$ & 0.88 & 0.93 \\
OH/OL (\%) & $55.39 \pm 3.44$ & 49.82 & 59.16 \\
OT/OL (\%) & $14.29 \pm 1.31$ & 12.09 & 15.22 \\
OT/OH (\%) & $25.79 \pm 1.58$ & 23.87 & 27.10 \\
Circularity & $23.48 \pm 1.83$ & 21.62 & 26.47 \\
Rectangularity & $0.72 \pm 0.02$ & 0.70 & 0.76 \\
\hline
\end{tabular}


Strongylura timucu (Walbaum 1792) - Plate 6

\begin{tabular}{ll} 
Maximum Size: & $610 \mathrm{~mm}$ (TL) (FROESE; PAULY, 2016). \\
$\begin{array}{l}\text { Distribution: } \\
\text { Habitat: }\end{array}$ & Western Atlantic, from Florida to Southern Brazil (FIGUEIREDO; MENEZES, 1978). \\
Diet: & Coastal areas and mangrove-lined lagoons (COLLETTE, 2002b). \\
Collection: & Feeds on small fishes (COLLETTE, 2002b). \\
Sample: & 3 otoliths from 3 fishes (TL ranging from 121 to $156 \mathrm{~mm}$ ). \\
\hline
\end{tabular}

Shape: elliptic to rectangular (67\%), elliptic. Anterior region: peaked. Posterior region: round. Dorsal edge: entire. Ventral edge: sinuate to entire. Profile: flattened. Rostrum and antirostrum orientation: in agreement. Rostrum: developed. Antirostrum: underdeveloped. Sulcus acusticus: position: median; orientation: horizontal; opening: ostial; morphology: heterosulcoid; colliculum: heteromorphic; ostium: funnel-like; cauda: tubular slightly curved.

The small number of otoliths examined did not permit the statistical analysis of the data but its morphometric characteristics are shown below:

\begin{tabular}{lccc}
\hline Shape indices & Mean \pm Sd & Minimum & Maximum \\
\hline OL/TL (\%) & $1.15 \pm 0.07$ & 1.12 & 1.23 \\
OH/OL (\%) & $55.32 \pm 2.39$ & 53.01 & 57.78 \\
OT/OL (\%) & $16.86 \pm 2.45$ & 14.37 & 19.26 \\
OT/OH (\%) & $30.44 \pm 3.87$ & 26.04 & 33.33 \\
Circularity & $15.49 \pm 0.22$ & 15.27 & 15.70 \\
Rectangularity & $0.76 \pm 0.02$ & 0.75 & 0.78 \\
\hline
\end{tabular}

\section{ORDER BERYCIFORMES}

\section{FAMILY BERYCIDAE}

Beryx splendens Lowe 1834 - Plate 7

\begin{tabular}{ll} 
Maximum Size: & $700 \mathrm{~mm}$ (TL) (SOMMER et al., 1996). \\
Distribution: & $\begin{array}{l}\text { Circumglobal. In the Western Atlantic, from Gulf of Maine to Uruguay (PAXTON, 1999; FROESE; PAULY, } \\
\text { 2016). }\end{array}$ \\
Habitat: & Benthopelagic, forming dense aggregations (MOORE, 2002a). \\
Diet: & Feeds on fishes and crustaceans (HORN et al., 2010). \\
Collection: & 236 otoliths from 236 fishes (TL ranging from 169 to $367 \mathrm{~mm}$ ). \\
Sample: & 29 right otoliths categorized into $8,20 \mathrm{~mm}$ classes (160 to $320 \mathrm{~mm})$. \\
\hline
\end{tabular}

Shape: trapezoidal. Anterior region: oblique (90\%), oblique to peaked. Posterior region: peaked-round (76\%), peaked, oblique-round, oblique. Dorsal edge: lobed (83\%), sinuate. Anterior ventral edge: lobed (90\%), entire, sinuate. Posterior ventral edge: lobed (79\%), sinuate. Central ventral edge: lobed. Profile: concave-convex (86\%), flattened. Rostrum and antirostrum orientation: does not apply (86\%), in agreement. Rostrum: developed (86\%), underdeveloped. Antirostrum: absent (86\%), underdeveloped. Pseudorostrum: absent. Pseudoantirostrum: absent. Sulcus acusticus: position: supramedian; orientation: ascending (93\%), horizontal; opening: ostio-caudal (66\%), ostial; morphology: heterosulcoid; colliculum: heteromorphic; ostium: bent-concave (93\%), tubular; cauda: tubular straight. 
Statistical differences $(p<0.05)$ within some length classes were obtained for dorsal, ventral and posterior ventral edges, anterior and posterior regions, rostrum and antirostrum development and orientation, sulcus acusticus opening and orientation, ostium morphology and profile. No differences were found along the fish development.

\begin{tabular}{lccc}
\hline Shape indices & Mean \pm Sd & Minimum & Maximum \\
\hline OL/TL (\%) & $4.58 \pm 0.29$ & 3.87 & 5.19 \\
OH/OL (\%) & $71.40 \pm 3.14$ & 66.18 & 77.88 \\
OT/OL (\%) & $11.27 \pm 1.45$ & 9.19 & 14.78 \\
OT/OH (\%) & $15.79 \pm 1.95$ & 12.40 & 20.29 \\
Circularity & $21.44 \pm 1.61$ & 18.39 & 24.29 \\
Rectangularity & $0.66 \pm 0.02$ & 0.61 & 0.72 \\
\hline
\end{tabular}

\section{FAMILY TRACHICHTHYIDAE}

\section{Hoplostethus occidentalis Woods 1973 - Plate 8}

\begin{tabular}{ll}
\hline $\begin{array}{l}\text { Maximum Size: } \\
\text { Distribution: }\end{array}$ & $250 \mathrm{~mm}$ (TL) (FIGUEIREDO; MENEZES, 1980). \\
& Western Atlantic, from Gulf of Mexico to Southern Brazil (FIGUEIREDO; MENEZES, 1980; MOORE, \\
& 2002b). \\
Habitat: & Benthopelagic, living along slopes at depth of 150 to $550 \mathrm{~m}$ (MOORE, 2002b). \\
Diet: & Feeds on small shrimps (FIGUEIREDO; MENEZES, 1980). \\
Collection: & 318 otoliths from 318 fishes (TL ranging from 93 to $245 \mathrm{~mm}$ ). \\
Sample: & 57 right otoliths categorized into $8,20 \mathrm{~mm}$ classes ( 80 to $260 \mathrm{~mm}$ ). \\
\hline
\end{tabular}

Shape: trapezoidal to irregular. Anterior region: oblique (86\%), oblique to peaked, flattened. Posterior region: oblique to angled (82\%), oblique, oblique to peaked, flattened to irregular. Dorsal edge: lobed (42\%), sinuate (33\%), lobed to sinuate, dentate to lobed. Anterior ventral edge: entire (47\%), sinuate (47\%), lobed. Posterior ventral edge: entire (58\%), sinuate, lobed. Central ventral edge: sinuate (81\%), entire. Profile: plane-convex. Rostrum and antirostrum orientation: in agreement. Rostrum: developed. Antirostrum: underdeveloped (72\%), developed. Pseudorostrum: absent. Pseudoantirostrum: absent. Sulcus acusticus: position: median; orientation: horizontal; opening: ostial; morphology: heterosulcoid; colliculum: heteromorphic; ostium: discoidal; cauda: elliptic.

Statistical differences $(p<0.05)$ within some length classes were obtained for dorsal, anterior ventral, posterior ventral and central ventral edges and anterior and posterior regions. Along the fish growth statistical differences were found for dorsal and ventral edges.

\begin{tabular}{lccc}
\hline Shape indices & Mean \pm Sd & Minimum & Maximum \\
\hline OL/TL (\%) & $6.23 \pm 0.27$ & 5.60 & 6.91 \\
OH/OL (\%) & $73.66 \pm 3.47$ & 66.58 & 81.35 \\
OT/OL (\%) & $16.92 \pm 1.63$ & 13.91 & 20.59 \\
OT/OH (\%) & $23.03 \pm 2.51$ & 17.57 & 27.61 \\
Circularity & $20.95 \pm 1.7$ & 18.27 & 25.86 \\
Rectangularity & $0.63 \pm 0.03$ & 0.55 & 0.69 \\
\hline
\end{tabular}




\section{Order ZEIFORMES}

\section{FAMILY GRAMMICOLEPIDIDAE}

Xenolepidichthys dalgleishi Gilchrist 1922 - Plate 9

\begin{tabular}{ll} 
Maximum Size: & $150 \mathrm{~mm}$ (TL) (HEEMSTRA, 2002), but attaining $260 \mathrm{~mm}$ in our collection. \\
$\begin{array}{l}\text { Distribution: } \\
\text { Western Atlantic, from Nova Scotia to Southern Brazil and Western Pacific (HEEMSTRA, 2002; FIGUEIRE- } \\
\text { Habitat: }\end{array}$ & DO et al., 2002). \\
Diet: & Benthic species, found on the continental slope (FROESE; PAULY, 2016). \\
Collection: & -- \\
Sample: & 1211 otoliths from 658 fishes (TL ranging from 68 to $260 \mathrm{~mm})$. \\
\hline
\end{tabular}

Shape: hour-glass. Anterior region: double-peaked. Posterior region: double-peaked. Dorsal edge: lobed (61\%), sinuate, lobed to sinuate. Ventral edge: sinuate (32\%), lobed to sinuate (24\%), lobed (24\%), sinuate to entire. Profile: concave-convex. Rostrum and antirostrum orientation: in agreement. Rostrum: developed. Antirostrum: developed. Pseudorostrum: developed. Pseudoantirostrum: developed. Sulcus acusticus: position: median; orientation: horizontal; opening: ostio-caudal; morphology: homosulcoid; colliculum: homomorphic; ostium: funnel-like; cauda: funnel-like.

Statistical differences $(p<0.05)$ within some length classes were obtained for dorsal and ventral edges. Along the fish's growth statistical differences were found for dorsal and ventral edges.

\begin{tabular}{lccc}
\hline Shape indices & Mean \pm Sd & Minimum & Maximum \\
\hline OL/TL (\%) & $1.78 \pm 0.22$ & 0.95 & 2.20 \\
OH/OL (\%) & $102.58 \pm 5.01$ & 90.91 & 112.98 \\
OT/OL (\%) & $23.33 \pm 2.73$ & 18.95 & 34.35 \\
OT/OH (\%) & $22.74 \pm 2.27$ & 18.75 & 30.41 \\
Circularity & $21.80 \pm 2.95$ & 1.85 & 27.02 \\
Rectangularity & $0.65 \pm 0.02$ & 0.59 & 0.70 \\
\hline
\end{tabular}

\section{FAMILY ZENIONTIDAE}

\section{Zenion hololepis (Goode \& Bean 1896) - Plate 10}

\begin{tabular}{ll} 
Maximum Size: & $173 \mathrm{~mm}$ (TL) (BERNARDES et al., 2005). \\
$\begin{array}{l}\text { Distribution: } \\
\text { Habitat: }\end{array}$ & Western Atlantic, from Gulf of Mexico to Southeastern Brazil (MENEZES et al., 2003). \\
Diet: & Found on tropical waters at depths between 300 to 500 meters (BERNARDES et al., 2005). \\
Collection: & -- \\
Sample: & 422 otoliths from 214 fishes (TL ranging from 56 to $173 \mathrm{~mm}$ ). \\
\hline
\end{tabular}

Shape: bullet-shaped (48\%), elliptic (37\%), discoidal, rectangular. Anterior region: round (59\%), angled-round, oblique. Posterior region: flattened (59\%), round, oblique. Dorsal edge: lobed to sinuate (37\%), lobed (37\%), sinuate to entire, dentate to sinuate. Ventral edge: entire (96\%), lobed, presenting an anterior and posterior projections. Profile: plane-convex. Rostrum and antirostrum orientation: does not apply. Rostrum: absent. Antirostrum: absent. Pseudorostrum: absent. Pseudoantirostrum: absent. Sulcus acusticus: position: median; orientation: ascending (70\%), horizontal; opening: mesial; morphology: pseudo-archaesulcoid; colliculum: homomorphic; ostium: round-oval; cauda: round-oval. 
Statistical differences $(p<0.05)$ within some length classes were obtained for shape, ventral edge, anterior and posterior regions and sulcus acusticus orientation. Along the fish growth statistical differences were found only for anterior region.

\begin{tabular}{lccc}
\hline Shape indices & Mean \pm Sd & Minimum & Maximum \\
\hline OL/TL (\%) & $3.59 \pm 0.66$ & 2.29 & 4.95 \\
OH/OL (\%) & $85.62 \pm 8.53$ & 71.84 & 101.01 \\
OT/OL (\%) & $20.68 \pm 1.57$ & 17.97 & 23.46 \\
OT/OH (\%) & $24.27 \pm 1.85$ & 21.50 & 29.78 \\
Circularity & $19.64 \pm 3.57$ & 15.28 & 28.40 \\
Rectangularity & $0.72 \pm 0.05$ & 0.62 & 0.79 \\
\hline
\end{tabular}

\section{ORDER SYNGNATHIFORMES}

\section{FAMIILY CENTRISCIDAE}

The otoliths of this family is clearly hour-glass, the pseudorostrum is always developed and pseudoantirostrum is frequently underdeveloped. The sulcus acusticus position is supramedial, the opening is ostio-caudal, morphology is homosulcoid, colliculum is homomorphic and ostium and cauda are always funnel-like.

Macroramphosus scolopax (Linnaeus 1758) - Plate 11

\begin{tabular}{ll} 
Maximum Size: & $200 \mathrm{~mm}$ (TL) (EHRICH, 1990). \\
Distribution: & Western Atlantic, from Gulf of Maine to Argentina; Eastern Atlantic, Mediterranean, and Indo-West Pacific \\
& (FROESE; PAULY, 2016). \\
Habitat: & Demersal; on the continental shelf over sand bottom (FROESE; PAULY, 2016). \\
Diet: & Feeds on invertebrates, mainly copepods (EHRICH, 1986). \\
Collection: & 15 otoliths from 10 fishes (TL ranging from 67 to $111 \mathrm{~mm})$. \\
Sample: & 3 left otoliths categorized into 3, $20 \mathrm{~mm}$ classes (60 to $100 \mathrm{~mm})$. \\
\hline
\end{tabular}

Shape: hour-glass. Anterior region: blunt. Posterior region: blunt. Dorsal edge: entire. Ventral edge: sinuate to entire. Profile: concave-convex. Rostrum and antirostrum orientation: in agreement. Rostrum: developed. Antirostrum: underdeveloped. Pseudorostrum: developed. Pseudoantirostrum: underdeveloped. Sulcus acusticus: position: supramedian; orientation: horizontal; opening: ostio-caudal; morphology: homosulcoid; colliculum: homomorphic; ostium: funnel-like; cauda: funnel-like.

The small number of otoliths examined did not permit the statistical analysis of the data but its morphometric characteristics are shown below:

\begin{tabular}{lccc}
\hline Shape índices & Mean \pm Sd & Minimum & Maximum \\
\hline OL/TL (\%) & $2.55 \pm 1.54$ & 1.05 & 4.13 \\
OH/OL (\%) & $95.37 \pm 9.44$ & 88.09 & 106.04 \\
OT/OL (\%) & $23.24 \pm 6.78$ & 18.49 & 31.00 \\
OT/OH (\%) & $24.69 \pm 8.27$ & 17.44 & 33.70 \\
Circularity & $15.70 \pm 0.51$ & 15.16 & 16.18 \\
Rectangularity & $0.67 \pm 0.03$ & 0.63 & 0.69 \\
\hline
\end{tabular}


Notopogon fernandezianus (Delfin 1899) - Plate 12

\begin{tabular}{|c|c|}
\hline Maximum Size: & 188 mm (TL) (FIGUEIREDO; MENEZES, 1980). \\
\hline Distribution: & $\begin{array}{l}\text { Southwest Atlantic, from Southern Brazil to Northern Argentina; Southeast Pacific (FIGUEIREDO; MENE- } \\
\text { ZES, 1980). }\end{array}$ \\
\hline Habitat: & Bathydemersal, up to $580 \mathrm{~m}$ depth on the continental shelf and slope (FROESE; PAULY, 2016). \\
\hline Diet: & -- \\
\hline Collection: & 21 otoliths from 11 fishes (TL ranging from 142 to $161 \mathrm{~mm}$ ). \\
\hline Sample: & 6 left otoliths categorized into 2, $20 \mathrm{~mm}$ classes (140 to $180 \mathrm{~mm}$ ). \\
\hline
\end{tabular}

Shape: hour-glass. Anterior region: notched (83\%), blunt. Posterior region: notched (67\%), blunt. Dorsal edge: entire. Ventral edge: sinuate (50\%), lobed to sinuate (50\%). Profile: concave-convex. Rostrum and antirostrum orientation: in agreement. Rostrum: developed. Antirostrum: underdeveloped. Pseudorostrum: developed. Pseudoantirostrum: underdeveloped (83\%), absent. Sulcus acusticus: position: supramedian; orientation: horizontal; opening: ostio-caudal; morphology: homosulcoid; colliculum: homomorphic; ostium: funnel-like; cauda: funnel-like.

The small number of otoliths examined did not permit the statistical analysis of the data but its morphometric characteristics are shown below:

\begin{tabular}{lccc}
\hline Shape indices & Mean \pm Sd & Minimum & Maximum \\
\hline OL/TL (\%) & $1.07 \pm 0.17$ & 0.93 & 1.34 \\
OH/OL (\%) & $102.44 \pm 5.58$ & 95.65 & 109.86 \\
OT/OL (\%) & $46.97 \pm 7.05$ & 38.79 & 57.63 \\
OT/OH (\%) & $46.09 \pm 8.28$ & 35.90 & 58.96 \\
Circularity & $19.34 \pm 1.71$ & 17.65 & 21.60 \\
Rectangularity & $0.61 \pm 0.03$ & 0.57 & 0.65 \\
\hline
\end{tabular}

\section{ORDER SCORPAENIFORMES}

\section{FAMILY TRIGLIDAE}

The otolith shape is frequently elliptic and pseudorostrum and pseudoantirostrum are always absent. The analyzed species present some variation in the sulcus acusticus opening which differs from other families.

Bellator brachychir (Regan 1914) - Plate 13

\begin{tabular}{ll} 
Maximum Size: & $160 \mathrm{~mm}$ (TL) (RICHARDS, 2002), but attaining 208 mm in our collection. \\
$\begin{array}{l}\text { Distribution: } \\
\text { Habitat: }\end{array}$ & Western Atlantic, from North Carolina to Uruguay (RICHARDS, 2002). \\
Diet: & Shallow waters, on bays, estuaries and mid-shelf (FROESE; PAULY, 2016). \\
Collection: & Feeds on crustaceans and small fishes (SÃO CLEMENTE et al., 2014). \\
Sample: & 83 otoliths from 45 fishes (TL ranging from 34 to $208 \mathrm{~mm})$. \\
\hline
\end{tabular}

Shape: elliptic (89\%), triangular to elliptic. Anterior region: blunt to peaked (32\%), peaked-round (26\%), angledround (21\%), peaked. Posterior region: flattened (32\%), peaked-round (21\%), round (21\%), peaked. Anterior dorsal edge: entire (89\%), sinuate. Posterior dorsal edge: does not apply (89\%), entire. Ventral edge: entire (53\%), sinuate (47\%). Profile: plane-convex. Rostrum and antirostrum orientation: does not apply (95\%), in agreement. Rostrum: developed. Antirostrum: absent (95\%), underdeveloped. Sulcus acusticus: position: median; orientation: horizontal; opening: ostial (89\%), pseudo-ostial; morphology: heterosulcoid; colliculum: heteromorphic; ostium: elliptic (74\%), funnel-like; cauda: round-oval. 
Statistical differences $(p<0.05)$ within some length classes were obtained for shape, anterior dorsal, posterior dorsal and ventral edges, anterior region, sulcus acusticus opening, antirostrum development, rostrum and antirostrum orientation and ostium morphology. Along the fish growth statistical differences were found for ventral edge, anterior and posterior regions.

\begin{tabular}{lccc}
\hline Shape índices & Mean \pm Sd & Minimum & Maximum \\
\hline OL/TL (\%) & $3.64 \pm 0.28$ & 3.22 & 4.21 \\
OH/OL (\%) & $66.51 \pm 5.17$ & 60.12 & 77.43 \\
OT/OL (\%) & $20.61 \pm 3.62$ & 17.14 & 29.10 \\
OT/OH (\%) & $31.23 \pm 6.59$ & 25.14 & 46.99 \\
Circularity & $14.49 \pm 0.5$ & 13.77 & 15.33 \\
Rectangularity & $0.71 \pm 0.02$ & 0.67 & 0.75 \\
\hline
\end{tabular}

Prionotus nudigula Ginsburg 1950 - Plate 14

\begin{tabular}{ll}
\hline Maximum Size: & $280 \mathrm{~mm}$ (TL) (FROESE; PAULY, 2016). \\
Distribution: & $\begin{array}{l}\text { Southwest Atlantic, from Southern Brazil to Argentina (MENEZES et al., 2003; FIGUEIREDO; MENEZES, } \\
\text { 1980). }\end{array}$ \\
Habitat: & Deep waters from 100 to $400 \mathrm{~m}$ depths (FIGUEIREDO et al., 2002). \\
Diet: & Mainly crustaceans (isopods and shrimps) (SÃO CLEMENTE et al., 2014). \\
Collection: & 627 otoliths from 326 fishes (TL ranging from 68 to $265 \mathrm{~mm}$ ). \\
Sample: & 95 left otoliths categorized into $11,20 \mathrm{~mm}$ classes (60 to $260 \mathrm{~mm}$ ). \\
\hline
\end{tabular}

Shape: elliptic. Anterior region: angled-round (82\%), peaked, peaked-round, round. Posterior region: angledround (77\%), peaked, round, peaked-round. Dorsal edge: sinuate (57\%), entire, lobed to sinuate, sinuate to entire. Ventral edge: sinuate (71\%), lobed to sinuate, entire, sinuate to entire. Profile: concave-convex. Rostrum and antirostrum orientation: in agreement (75\%), does not apply. Rostrum: developed (87\%), underdeveloped. Antirostrum: underdeveloped (74\%), absent, developed. Sulcus acusticus: position: median; orientation: horizontal; opening: ostial (78\%), para-ostial, pseudo-ostial, ostio-caudal; morphology: heterosulcoid; colliculum: heteromorphic; ostium: funnel-like (56\%), elliptic; cauda: tubular markedly curved (64\%), tubular strongly curved, round-oval, elliptic.

Significant differences $(p<0.05)$ were obtained within some length classes for dorsal and ventral edges, anterior and posterior regions, sulcus acusticus opening and position, ostium and cauda morphology and rostrum and antirostrum development and orientation. Along the fish development statistical differences were found for dorsal and ventral edges, cauda morphology, rostrum and antirostrum development and orientation.

\begin{tabular}{lccc}
\hline Shape indices & Mean \pm Sd & Minimum & Maximum \\
\hline OL/TL (\%) & $3.47 \pm 1.43$ & 1.85 & 7.47 \\
OH/OL (\%) & $63.50 \pm 3.55$ & 54.24 & 70.36 \\
OT/OL (\%) & $17.29 \pm 1.91$ & 11.72 & 22.70 \\
OT/OH (\%) & $27.30 \pm 3.2$ & 18.51 & 34.93 \\
Circularity & $14.65 \pm 0.44$ & 13.87 & 16.60 \\
Rectangularity & $0.73 \pm 0.02$ & 0.68 & 0.77 \\
\hline
\end{tabular}


Prionotus punctatus (Bloch 1793) - Plate 15

\begin{tabular}{|c|c|}
\hline Maximum Size: & $450 \mathrm{~mm}$ (TL) (FROESE; PAULY, 2016). \\
\hline Distribution: & Western Atlantic, from Belize and Central America to Argentina (FIGUEIREDO et al., 2002). \\
\hline Habitat: & $\begin{array}{l}\text { Found on sand or muddy bottoms of the continental shelves from } 5 \text { to } 200 \mathrm{~m} \text { depth (CARVALHO-FILHO, } \\
\text { 1992). }\end{array}$ \\
\hline Diet: & Nocturnal habits, feeds mainly on crustaceans (SOARES; APELBAUM, 1994). \\
\hline Collection: & 736 otoliths from 394 fishes (TL ranging from 7 to $422 \mathrm{~mm}$ ). \\
\hline Sample: & 59 left otoliths categorized into $14,20 \mathrm{~mm}$ classes (20 to $380 \mathrm{~mm}$ ). \\
\hline
\end{tabular}

Shape: elliptic. Anterior region: peaked (73\%), angled-round, peaked-round. Posterior region: round (51\%), angledround, peaked, flattened. Dorsal edge: lobed to sinuate (44\%), dentate to lobed, lobed, sinuate to entire. Ventral edge: lobed to sinuate (34\%), lobed (31\%), dentate to lobed (27\%), sinuate to entire. Profile: concave-convex. Rostrum and antirostrum orientation: does not apply (97\%), in agreement. Rostrum: developed. Antirostrum: absent (97\%), underdeveloped. Sulcus acusticus: position: median; orientation: horizontal; opening: pseudo-ostial (71\%), ostial; morphology: heterosulcoid; colliculum: heteromorphic; ostium: elliptic (53\%), tubular, funnel-like; cauda: tubular strongly curved (71\%), tubular markedly curved.

Significant differences $(p<0.05)$ were obtained within some length classes for dorsal and ventral edges, anterior and posterior regions, sulcus acusticus opening, ostium and cauda morphology, antirostrum development and rostrum and antirostrum orientation. Along the fish development statistical differences were found only for the ostium morphology.

\begin{tabular}{lccc}
\hline Shape índices & Mean \pm Sd & Minimum & Maximum \\
\hline OL/TL (\%) & $3.23 \pm 0.39$ & 2.36 & 4.19 \\
OH/OL (\%) & $66.15 \pm 2.36$ & 59.61 & 71.02 \\
OT/OL (\%) & $14.84 \pm 2.23$ & 10.17 & 20.23 \\
OT/OH (\%) & $22.43 \pm 3.27$ & 16.34 & 30.17 \\
Circularity & $18.66 \pm 2.29$ & 14.30 & 24.40 \\
Rectangularity & $0.69 \pm 0.02$ & 0.66 & 0.73 \\
\hline
\end{tabular}

\section{FAMILY DACTYLOPTERIDAE}

Dactylopterus volitans (Linnaeus 1758) - Plate 16

\begin{tabular}{ll} 
Maximum Size: & $500 \mathrm{~mm}$ (TL) (FROESE; PAULY, 2016). \\
$\begin{array}{l}\text { Distribution: } \\
\text { Habitat: }\end{array}$ & Eastern and Western Atlantic, from Bermuda to Argentina (FIGUEIREDO; MENEZES, 1980). \\
& Found on sand and mud bottoms near rocky areas and reefs, exploring the bottom with the pectoral fins (CAR- \\
Diet: & VALHO-FILHO, 1992). \\
Collection: & Feeds mainly on crustaceans, mollusks and small fishes (BERNARDES et al., 2005). \\
Sample: & 2571 otoliths from 1426 fishes (TL ranging from 65 to $292 \mathrm{~mm}$ ). \\
\hline
\end{tabular}

Shape: oval (80\%), discoidal, elliptic, hour-glass. Anterior region: double-peaked (62\%), peaked-round, blunt, angled. Posterior region: oblique-round (50\%), round (44\%), blunt, flattened. Dorsal edge: entire (94\%), sinuate, lobed, crenate. Ventral edge: entire (42\%), sinuate, crenate, lobed. Profile: plane-convex. Rostrum and antirostrum orientation: in agreement. Rostrum: developed (67\%), underdeveloped. Antirostrum: underdeveloped (51\%), developed (49\%). Pseudorostrum: absent (98\%), underdeveloped, developed. Pseudoantirostrum: absent (98\%), underdeveloped, developed. Sulcus acusticus: position: median; orientation: horizontal; opening: ostial (95\%), ostio-caudal; morphology: heterosulcoid; colliculum: heteromorphic; ostium: funnel-like; cauda: tubular markedly curved (90\%), tubular strongly curved, round-oval. 
Significant differences $(p<0.05)$ were obtained within some length classes for shape, dorsal and ventral edges, anterior and posterior regions, sulcus acusticus opening, cauda morphology and rostrum, antirostrum, pseudorostrum and pseudoantirostrum development. Along the otoliths growth statistical differences were found for ventral edge, anterior and posterior regions and rostrum and antirostrum development.

\begin{tabular}{lccc}
\hline Shape indices & Mean \pm Sd & Minimum & Maximum \\
\hline OL/TL (\%) & $1.15 \pm 0.19$ & 0.77 & 1.65 \\
OH/OL (\%) & $77.04 \pm 5.8$ & 63.27 & 95.24 \\
OT/OL (\%) & $31.82 \pm 3.7$ & 25.28 & 44.44 \\
OT/OH (\%) & $41.30 \pm 3.58$ & 33.85 & 52.83 \\
Circularity & $16.66 \pm 2.5$ & 13.38 & 25.13 \\
Rectangularity & $0.71 \pm 0.03$ & 0.62 & 0.80 \\
\hline
\end{tabular}

\section{FAMIILY SEBASTIDAE}

Helicolenus lahillei Norman 1937 - Plate 17

\begin{tabular}{ll}
\hline Maximum Size: & $462 \mathrm{~mm}$ (TL) (BERNARDES et al., 2005). \\
Distribution: & Southwest Atlantic, from Rio Grande do Sul to Argentina (MENEZES et al., 2003). \\
Habitat: & Demersal species, found on deep waters (FIGUEIREDO et al., 2002). \\
Diet: & Feeds on crustaceans, mollusks and small fishes (BERNARDES et al., 2005). \\
Collection: & 348 otoliths from 339 fishes (TL ranging from 61 to $462 \mathrm{~mm})$. \\
Sample: & 96 left otoliths categorized into $18,20 \mathrm{~mm}$ classes (60 to $460 \mathrm{~mm}$ ). \\
\hline
\end{tabular}

Shape: elliptic (79\%), fusiform, elliptic to lanceolated, fusiform to lanceolated. Anterior region: peaked (91\%), lanceolated. Posterior region: round (32\%), oblique-round (26\%), flattened, peaked. Dorsal edge: entire (45\%), sinuate (41\%), lobed, lobed to sinuate. Ventral edge: sinuate (39\%), lobed to sinuate (35\%), serrate to entire, entire. Profile: concave-convex. Rostrum and antirostrum orientation: in agreement (60\%), does not apply. Rostrum: developed (98\%), absent. Antirostrum: underdeveloped (60\%), absent. Pseudorostrum: absent. Pseudoantirostrum: absent. Sulcus acusticus: position: inframedian (67\%), median; orientation: horizontal; opening: ostial (93\%), para-ostial, pseudo-ostial; morphology: heterosulcoid; colliculum: heteromorphic; ostium: funnel-like (90\%), elliptic; cauda: tubular straight (52\%), tubular slightly curved (48\%).

Significant differences $(p<0.05)$ were obtained within some length classes for shape, dorsal and ventral edges, anterior and posterior regions, sulcus acusticus opening and position, ostium and cauda morphology and rostrum development. Along the fish development statistical differences were found for dorsal and ventral edges, posterior region, sulcus acusticus position and cauda morphology.

\begin{tabular}{lccc}
\hline Shape indices & Mean \pm Sd & Minimum & Maximum \\
\hline OL/TL (\%) & $4.08 \pm 0.5$ & 2.78 & 5.38 \\
OH/OL (\%) & $53.44 \pm 2.69$ & 46.35 & 60.19 \\
OT/OL (\%) & $14.64 \pm 2.2$ & 11.62 & 23.15 \\
OT/OH (\%) & $27.46 \pm 4.4$ & 21.72 & 44.23 \\
Circularity & $18.07 \pm 1.73$ & 15.82 & 24.49 \\
Rectangularity & $0.66 \pm 0.02$ & 0.59 & 0.70 \\
\hline
\end{tabular}




\section{FAMILY PERISTEDIIDAE}

Peristedion altipinne (Regan 1903) - Plate 18

\begin{tabular}{ll}
\hline Maximum Size: & $260 \mathrm{~mm}$ (TL) (BERNARDES et al., 2005). \\
$\begin{array}{l}\text { Distribution: } \\
\text { Habitat: }\end{array}$ & Southwest Atlantic, from Rio de Janeiro to Rio Grande do Sul (FIGUEIREDO et al., 2002). \\
Diet: & Inhabits deep waters of the continental shelf, between 100 to $200 \mathrm{~m}$ depth (BERNARDES et al., 2005). \\
Collection: & -- \\
Sample: & 339 otoliths from 188 fishes (TL ranging from 112 to $258 \mathrm{~mm}$ ). \\
\hline
\end{tabular}

Shape: elliptic (56\%), oval. Anterior region: double-peaked (33\%), peaked, blunt, round. Posterior region: round (35\%), angled-round (33\%), angled, oblique. Dorsal edge: sinuate (76\%), entire, lobed to sinuate. Ventral edge: sinuate (82\%), entire, lobed to sinuate. Profile: concave-convex. Rostrum and antirostrum orientation: in agreement (69\%), does not apply. Rostrum: underdeveloped (65\%), developed, absent. Antirostrum: underdeveloped (56\%), absent, developed. Pseudorostrum: absent. Pseudoantirostrum: absent (98\%), underdeveloped. Sulcus acusticus: position: median (69\%), inframedian; orientation: horizontal; opening: ostial (85\%), pseudo-ostial, para-ostial, ostio-caudal morphology: heterosulcoid; colliculum: heteromorphic; ostium: elliptic (95\%), funnel-like; cauda: tubular straight (93\%), tubular strongly curved, tubular slightly curved.

Significant differences $(p<0.05)$ were obtained within some length classes for shape, dorsal and ventral edges, anterior and posterior regions, sulcus acusticus opening and position, ostium and cauda morphology and rostrum and pseudoantirostrum development. Along the otolith growth statistical differences were found for shape, anterior region and sulcus acusticus position.

\begin{tabular}{lccc}
\hline Shape indices & Mean \pm Sd & Minimum & Maximum \\
\hline OL/TL (\%) & $1.79 \pm 0.16$ & 1.49 & 2.38 \\
OH/OL (\%) & $73.32 \pm 5.38$ & 60.13 & 84.15 \\
OT/OL (\%) & $20.47 \pm 2.00$ & 17.13 & 25.88 \\
OT/OH (\%) & $28.01 \pm 2.9$ & 22.83 & 35.38 \\
Circularity & $14.96 \pm 1.06$ & 13.44 & 18.67 \\
Rectangularity & $0.71 \pm 0.02$ & 0.65 & 0.76 \\
\hline
\end{tabular}

\section{FAMIILY SCORPAENIDAE}

The two species of this family are very different from each other and presented no common features. The pseudorostrum and pseudoantirostrum are always absent.

Pontinus rathbuni Goode \& Bean 1896 - Plate 19

\begin{tabular}{ll} 
Maximum Size: & $250 \mathrm{~mm}$ (TL) (FROESE; PAULY, 2016). \\
Distribution: & Western Atlantic, from Rio de Janeiro to Southern Brazil (MENEZES et al., 2003). FIGUEIREDO \& \\
& MENEZES (1980) treat the species as a synonym of Pontinus corallinus. \\
Habitat: & Demersal species, found at depths between 90 to 215 meters (BERNARDES et al., 2005) \\
Diet: & -- \\
Collection: & 2 otoliths from 1 fish (TL ranging $97 \mathrm{~mm})$. \\
Sample: & 1 left otolith categorized into $1,20 \mathrm{~mm}$ classes $(97 \mathrm{~mm})$. \\
\hline
\end{tabular}


Shape: elliptic. Anterior region: peaked-round. Posterior region: peaked. Dorsal edge: entire. Ventral edge: sinuate to entire. Profile: plane-convex. Rostrum and antirostrum orientation: in agreement. Rostrum: developed. Antirostrum: underdeveloped. Sulcus acusticus: position: median; orientation: horizontal; opening: para-ostial; morphology: heterosulcoid; colliculum: heteromorphic; ostium: elliptic; cauda: tubular strongly curved.

The small number of otoliths examined did not allow the statistical analysis of the data but its morphometric characteristics are shown below:

\begin{tabular}{lccc}
\hline Shape indices & Mean \pm Sd & Minimum & Maximum \\
\hline OL/TL (\%) & $5.43 \pm 0$ & 5.43 & 5.43 \\
OH/OL (\%) & $54.27 \pm 0$ & 54.27 & 54.27 \\
OT/OL (\%) & $19.54 \pm 0$ & 19.54 & 19.54 \\
OT/OH (\%) & $36.01 \pm 0$ & 36.01 & 36.01 \\
Circularity & $16.02 \pm 0$ & 16.02 & 16.02 \\
Rectangularity & $0.68 \pm 0$ & 0.68 & 0.68 \\
\hline
\end{tabular}

Scorpaena dispar Longley \& Hildebrand 1940 - Plate 20

\begin{tabular}{ll}
\hline $\begin{array}{l}\text { Maximum Size: } \\
\text { Distribution: }\end{array}$ & $272 \mathrm{~mm}$ (TL) (FIGUEIREDO; MENEZES, 1980). \\
Habitat: & Western Atlantic, from Florida to Rio de Janeiro, Brazil (MENEZES et al., 2003). \\
Diet: & Inhabits offshore waters at depths between 30 to 120 meters (CARVALHO-FILHO, 1992). \\
Collection: & -- \\
Sample: & 4 otoliths from 2 fishes (TL ranging from 38 to $129 \mathrm{~mm}$ ). \\
\hline
\end{tabular}

Shape: elliptic. Anterior region: peaked. Posterior region: round (50\%), peaked (50\%). Dorsal edge: sinuate to entire. Ventral edge: entire (50\%), sinuate to entire (50\%). Profile: concave-convex (50\%), biconvex (50\%). Rostrum and antirostrum orientation: does not apply. Rostrum: developed. Antirostrum: absent. Sulcus acusticus: position: median; orientation; horizontal; opening: ostial; morphology: heterosulcoid; colliculum: heteromorphic; ostium: elliptic; cauda: tubular slightly curved (50\%), round-oval (50\%).

The small number of otoliths examined did not permit the statistical analysis of the data but its morphometric characteristics are shown below:

\begin{tabular}{lccc}
\hline Shape índices & Mean \pm Sd & Minimum & Maximum \\
\hline OL/TL (\%) & $5.97 \pm 0.57$ & 5.57 & 6.37 \\
OH/OL (\%) & $53.58 \pm 5.46$ & 49.72 & 57.44 \\
OT/OL (\%) & $22.13 \pm 8.45$ & 16.16 & 28.10 \\
OT/OH (\%) & $40.71 \pm 11.62$ & 32.49 & 48.92 \\
Circularity & $15.97 \pm 1.25$ & 15.09 & 16.85 \\
Rectangularity & $0.68 \pm 0.03$ & 0.66 & 0.70 \\
\hline
\end{tabular}

\section{FAMILY SETARCHIDAE}

Setarches guentheri Johnson 1862 - Plate 21

\begin{tabular}{ll}
\hline $\begin{array}{l}\text { Maximum Size: } \\
\text { Distribution: }\end{array}$ & $250 \mathrm{~mm}$ (TL) (FROESE; PAULY, 2016), but attaining $255 \mathrm{~mm}$ in our collection. \\
& $\begin{array}{l}\text { Eastern and Western Atlantic, from USA to Southern Brazil. Also occurs in the Indo-West and Eastern Pacific } \\
\text { (FIGUEIREDO et al., 2002; MENEZES et al., 2003). }\end{array}$ \\
Habitat: & Inhabits offshore waters living on or near to the bottom (FIGUEIREDO et al., 2002). \\
Diet: & Feeds mainly on bathypelagic crustaceans (FROESE; PAULY, 2016). \\
Collection: & 944 otoliths from 477 fishes (TL ranging from 43 to $255 \mathrm{~mm}$ ). \\
Sample: & 100 left otoliths categorized into $11,20 \mathrm{~mm}$ classes ( 40 to $240 \mathrm{~mm}$ ). \\
\hline
\end{tabular}


Shape: elliptic (65\%), elliptic to lanceolated, fusiform to lanceolated, elliptic to cuneiform. Anterior region: peaked (50\%), lanceolated-round, peaked-round, lanceolated. Posterior region: round (74\%), angled-round, flattened, double-peaked. Dorsal edge: lobed (36\%), lobed to sinuate (34\%), sinuate (27\%), entire. Ventral edge: sinuate (55\%), entire, lobed to sinuate, sinuate to entire. Profile: flattened (64\%), plane-convex. Rostrum and antirostrum orientation: in agreement (84\%), does not apply. Rostrum: developed. Antirostrum: underdeveloped (67\%), developed, absent. Pseudorostrum: absent (99\%), underdeveloped. Pseudoantirostrum: absent (99\%), underdeveloped. Sulcus acusticus: position: median; orientation: horizontal; opening: ostial (99\%), ostio-caudal; morphology: pseudo-archaesulcoid; colliculum: heteromorphic; ostium: funnel-like; cauda: elliptic.

Significant differences $(p<0.05)$ were obtained within some length classes for shape, profile, dorsal and ventral edges, anterior and posterior regions, sulcus acusticus opening, ostium and cauda morphology, antirostrum, pseudorostrum and pseudoantirostrum development and rostrum and antirostrum orientation. Along the fish development statistical differences were found for shape, profile, dorsal and ventral edges and anterior region.

\begin{tabular}{lccc}
\hline Shape indices & Mean \pm Sd & Minimum & Maximum \\
\hline OL/TL (\%) & $4.83 \pm 0.38$ & 3.05 & 5.88 \\
OH/OL (\%) & $61.51 \pm 5.96$ & 34.38 & 73.47 \\
OT/OL (\%) & $18.59 \pm 2.57$ & 13.76 & 25.44 \\
OT/OH (\%) & $30.32 \pm 3.9$ & 23.42 & 58.04 \\
Circularity & $17.73 \pm 2.33$ & 4.61 & 23.95 \\
Rectangularity & $0.67 \pm 0.06$ & 0.62 & 0.72 \\
\hline
\end{tabular}

\section{ORDER TETRAODONTIFORMES}

\section{FAMILY DIODONTIDAE}

Chilomycterus spinosus (Linnaeus 1758) - Plate 22

\begin{tabular}{ll}
\hline Maximum Size: & $350 \mathrm{~mm}$ (TL) (BERNARDES et al., 2005). \\
Distribution: & Southwest Atlantic, from Bahia to Argentina (MENEZES et al., 2003; FIGUEIREDO et al., 2002). \\
Habitat: & Found on estuarine waters to $190 \mathrm{~m}$ depth (FIGUEIREDO; MENEZES, 2000). \\
Diet: & Feeds mainly on invertebrates (bivalves, barnacles, polychaetes) (ALMEIDA-SILVA et al., 2015). \\
Collection: & 150 otoliths from 86 fishes (TL ranging from 40 to $190 \mathrm{~mm}$ ). \\
Sample: & 13 left otoliths categorized into 3, $20 \mathrm{~mm}$ classes ( 40 to $80 \mathrm{~mm}$ ). \\
\hline
\end{tabular}

Shape: discoidal (58\%), tall, irregular. Anterior region: round (69\%), double-peaked, notched, flattened. Posterior region: round. Dorsal edge: entire (62\%), sinuate. Ventral edge: entire (62\%), sinuate. Profile: plane-convex. Rostrum and antirostrum orientation: does not apply (92\%), in agreement. Rostrum: absent. Antirostrum: absent (92\%), underdeveloped. Pseudorostrum: absent. Pseudoantirostrum: absent. Sulcus acusticus: position: inframedian; orientation: horizontal; opening: ostio-caudal (92\%), ostial; morphology: pseudo-archaesulcoid; colliculum: monomorphic; ostium: tubular (54\%), funnel-like (46\%); cauda: tubular markedly curved (62\%), tubular straight.

Significant differences $(p<0.05)$ were obtained within some length classes for shape, dorsal and ventral edges, anterior region, sulcus acusticus opening, antirostrum development and rostrum and antirostrum orientation. Along the otolith growth statistical differences were found for dorsal and ventral edges.

\begin{tabular}{lccc}
\hline Shape indices & Mean \pm Sd & Minimum & Maximum \\
\hline OL/TL (\%) & $0.85 \pm 0.16$ & 0.62 & 1.19 \\
OH/OL (\%) & $117.93 \pm 10.21$ & 96.10 & 135.71 \\
OT/OL (\%) & $44.61 \pm 6.19$ & 33.33 & 54.76 \\
OT/OH (\%) & $37.97 \pm 5.49$ & 29.41 & 47.92 \\
Circularity & $14.49 \pm 1.56$ & 13.16 & 17.87 \\
Rectangularity & $0.76 \pm 0.06$ & 0.68 & 0.85 \\
\hline
\end{tabular}


Diodon holocanthus Linnaeus 1758 - Plate 23

\begin{tabular}{ll} 
Maximum Size: & $500 \mathrm{~mm}(\mathrm{TL})$ (CARVALHO-FILHO, 1992). \\
$\begin{array}{l}\text { Distribution: } \\
\text { Habitat: }\end{array}$ & Circumtropical. In the Western Atlantic, from Florida to Southern Brazil (MENEZES et al., 2003). \\
& Inhabits algae bottoms and estuarine areas, also found in areas with rocky bottoms (CARVALHO-FILHO, \\
1992). & Feeds mainly on bivalves, gastropods and crustaceans (HUIZAR; CARRARA, 2000). \\
Collection: & 4 otoliths from 2 fishes (TL ranging from 106 to $111 \mathrm{~mm})$. \\
Sample: & 2 left otoliths categorized into $1,20 \mathrm{~mm}$ classes $(100 \mathrm{~mm})$. \\
\hline
\end{tabular}

Shape: tall. Anterior region: peaked. Posterior region: round. Dorsal edge: sinuate (50\%), lobed (50\%). Ventral edge: sinuate (50\%), entire (50\%). Profile: concave-convex. Rostrum and antirostrum orientation: in agreement. Rostrum: developed. Antirostrum: underdeveloped. Pseudorostrum: absent. Pseudoantirostrum: absent. Pseudorostrum: absent. Pseudoantirostrum: absent. Sulcus acusticus: position: supramedian; orientation: horizontal (50\%), descending (50\%); opening: ostial; morphology: heterosulcoid; colliculum: heteromorphic; ostium: funnel-like; cauda round-oval.

The small number of otoliths examined did not allow the statistical analysis of the data but its morphometric characteristics are shown below:

\begin{tabular}{lccc}
\hline Shape indices & Mean \pm Sd & Minimum & Maximum \\
\hline OL/TL (\%) & $0.70 \pm 0.05$ & 0.67 & 0.74 \\
OH/OL (\%) & $131.84 \pm 14.21$ & 121.79 & 141.89 \\
OT/OL (\%) & $54.02 \pm 3.87$ & 51.28 & 56.76 \\
OT/OH (\%) & $41.05 \pm 1.49$ & 40.00 & 42.11 \\
Circularity & $15.22 \pm 0.54$ & 14.83 & 15.60 \\
Rectangularity & $0.76 \pm 0.05$ & 0.73 & 0.80 \\
\hline
\end{tabular}

\section{FAMILY TETRAODONTIDAE}

The otolith of this family is clearly hour-glass the sulcus acusticus position is medial, the orientation is horizontal, morphology is homosulcoid, colliculum is homomorphic and ostium and cauda are always funnel-like.

Lagocephalus laevigatus (Linnaeus 1766) - Plate 24

\begin{tabular}{ll}
\hline Maximum Size: & $1000 \mathrm{~mm}$ (TL) (FIGUEIREDO et al., 2002). \\
Distribution: & Eastern and Western Atlantic, from New England to Argentina (FIGUEIREDO; MENEZES, 2000; \\
& FIGUEIREDO et al., 2002). \\
Habitat: & $\begin{array}{l}\text { Juveniles inhabit inshore areas over sand or mud bottoms; adults are pelagic found on continental margins } \\
\text { (CARVALHO-FILHO, 1992). }\end{array}$ \\
Diet: & Feeds mostly on crustaceans and fishes (DENADAI et al., 2012). \\
Collection: & 1 otolith from 1 fish (TL ranging $144 \mathrm{~mm})$. \\
Sample: & 1 left otolith categorized into $1,20 \mathrm{~mm}$ classes $(140 \mathrm{~mm})$. \\
\hline
\end{tabular}

Shape: hour-glass. Anterior region: notched. Posterior region: notched. Dorsal edge: lobed to sinuate. Ventral edge: lobed to sinuate. Profile: concaveconvex. Rostrum and antirostrum orientation: in agreement. Rostrum: developed. Antirostrum: developed. Pseudorostrum: underdeveloped. Pseudoantirostrum: underdeveloped. Sulcus acusticus: position: median; orientation: horizontal; opening: ostio-caudal; morphology: homosulcoid; colliculum: homomorphic; ostium: funnel-like; cauda: funnel-like.

The small number of otoliths examined did not permit the statistical analysis of the data but it morphometric characteristics are shown below: 


\begin{tabular}{lccc}
\hline Shape indices & Mean \pm Sd & Minimum & Maximum \\
\hline OL/TL (\%) & $0.53 \pm 0$ & 0.53 & 0.53 \\
OH/OL (\%) & $103.95 \pm 0$ & 103.95 & 103.95 \\
OT/OL (\%) & $32.89 \pm 0$ & 32.89 & 32.89 \\
OT/OH (\%) & $31.65 \pm 0$ & 31.65 & 31.65 \\
Circularity & $21.27 \pm 0$ & 21.27 & 21.27 \\
Rectangularity & $0.65 \pm 0$ & 0.65 & 0.65 \\
\hline
\end{tabular}

Sphoeroides greeleyi Gilbert 1900 - Plate 25

\begin{tabular}{ll}
\hline Maximum Size: & $180 \mathrm{~mm}$ (TL) (CERVIGÓN et al., 1992). \\
$\begin{array}{l}\text { Distribution: } \\
\text { Habitat: }\end{array}$ & Western Atlantic, from Honduras to Southern Brazil (MENEZES et al., 2003). \\
Diet: & Found on bays and estuarine waters over soft bottoms (FIGUEIREDO; MENEZES, 2000). \\
Collection: & Feeds on marine invertebrates (FIGUEIREDO; MENEZES, 2000). \\
Sample: & 119 otoliths from 69 fishes (TL ranging from 51 to $145 \mathrm{~mm})$. \\
& 23 left otoliths categorized into $5,20 \mathrm{~mm}$ classes (60 to $140 \mathrm{~mm}$ ).
\end{tabular}

Shape: hour-glass (87\%), trapezoidal to elliptic. Anterior region: double-peaked-round (52\%), double-peaked (43\%), angled-round. Posterior region: double-peaked-round (61\%), oblique to angled, oblique-round. Dorsal edge: entire (61\%), lobed. Ventral edge: entire (61\%), sinuate to entire. Profile: planeconvex. Rostrum and antirostrum orientation: in agreement. Rostrum: developed (96\%), underdeveloped. Antirostrum: underdeveloped (74\%), developed. Pseudorostrum: developed (74\%), underdeveloped, absent. Pseudoantirostrum: underdeveloped (74\%), absent. Sulcus acusticus: position: median; orientation: horizontal; opening: ostio-caudal; morphology: homosulcoid; colliculum: homomorphic; ostium: funnel-like; cauda: funnel-like.

Significant differences $(p<0.05)$ were obtained within some length classes for shape, dorsal and ventral edges, anterior and posterior regions and rostrum, antirostrum, pseudorostrum and pseudoantirostrum development. Along the fish's development statistical differences were found for dorsal and ventral edges, anterior and posterior regions and pseudoantirostrum development.

\begin{tabular}{lccc}
\hline Shape índices & Mean \pm Sd & Minimum & Maximum \\
\hline OL/TL (\%) & $1.05 \pm 0.12$ & 0.74 & 1.26 \\
OH/OL (\%) & $89.63 \pm 5.72$ & 80.95 & 105.06 \\
OT/OL (\%) & $39.18 \pm 4.43$ & 32.11 & 49.37 \\
OT/OH (\%) & $43.69 \pm 3.83$ & 36.08 & 51.76 \\
Circularity & $17.91 \pm 4.63$ & 14.00 & 33.33 \\
Rectangularity & $0.65 \pm 0.05$ & 0.52 & 0.71 \\
\hline
\end{tabular}

Sphoeroides testudineus (Linnaeus 1758) - Plate 26

\begin{tabular}{|c|c|}
\hline Maximum Size: & 388 mm (TL) (FROESE; PAULY, 2016). \\
\hline Distribution: & Western Atlantic, from New Jersey to Southern Brazil (MENEZES et al., 2003). \\
\hline Habitat: & $\begin{array}{l}\text { Commonly found in bays and protected coastal waters being rare or absent in coral reefs (CARVALHO-FILHO, } \\
\text { 1992; FIGUEIREDO; MENEZES, 2000). }\end{array}$ \\
\hline Diet: & $\begin{array}{l}\text { Feeds mainly on bivalves, gastropods and other benthic invertebrates (CHI-ESPÍNOLA; VEGA-CENDEJAS, } \\
\text { 2013). }\end{array}$ \\
\hline Collection: & 9 otoliths from 5 fishes (TL ranging from 79 to $232 \mathrm{~mm}$ ). \\
\hline Sample: & 2 left otoliths categorized into $2,20 \mathrm{~mm}$ classes ( 80 to $200 \mathrm{~mm}$ ). \\
\hline
\end{tabular}


Shape: hour-glass. Anterior region: blunt to peaked (50\%), double-peaked (50\%). Posterior region: blunt-round. Dorsal edge: sinuate to entire. Ventral edge: entire (50\%), sinuate to entire (50\%). Profile: plane-convex. Rostrum and antirostrum orientation: in agreement. Rostrum: developed. Antirostrum: underdeveloped (50\%), developed (50\%). Pseudorostrum: underdeveloped. Pseudoantirostrum: underdeveloped. Sulcus acusticus: position: median; orientation: horizontal; opening: ostio-caudal; morphology: homosulcoid; colliculum: homomorphic; ostium: funnel-like; cauda: funnel-like.

The small number of otoliths examined did not allow the statistical analysis of the data but its morphometric characteristics are shown below:

\begin{tabular}{lccc}
\hline Shape indices & Mean \pm Sd & Minimum & Maximum \\
\hline OL/TL (\%) & $1.10 \pm 0.49$ & 0.75 & 1.45 \\
OH/OL (\%) & $87.28 \pm 3.85$ & 84.56 & 90.00 \\
OT/OL (\%) & $32.46 \pm 12.32$ & 23.75 & 41.18 \\
OT/OH (\%) & $37.54 \pm 15.77$ & 26.39 & 48.70 \\
Circularity & $16.48 \pm 1.13$ & 15.68 & 17.28 \\
Rectangularity & $0.64 \pm 0$ & 0.64 & 0.64 \\
\hline
\end{tabular}

\section{FAMILY MONACANTHIDAE}

Stephanolepis hispidus (Linnaeus 1766) - Plate 27

\begin{tabular}{|c|c|}
\hline Maximum Size: & $300 \mathrm{~mm}$ (TL) (CARVALHO-FILHO, 1992). \\
\hline Distribution: & $\begin{array}{l}\text { Eastern and Western Atlantic, from Nova Scotia to Uruguay (FIGUEIREDO et al., 2002; MENEZES et al., } \\
\text { 2003). }\end{array}$ \\
\hline Habitat: & Found on coastal waters especially on rocky, reef and algae bottoms (CARVALHO-FILHO, 1992). \\
\hline Diet: & Feeds on benthic invertebrates (FROESE; PAULY, 2016). \\
\hline Collection: & 22 otoliths from 13 fishes (TL ranging from 36 to $195 \mathrm{~mm}$ ). \\
\hline Sample: & 4 left otoliths categorized into $3,20 \mathrm{~mm}$ classes ( 20 to $180 \mathrm{~mm}$ ). \\
\hline
\end{tabular}

Shape: hour-glass. Anterior region: notched (75\%), blunt to angled. Posterior region: notched (50\%), blunt (50\%). Dorsal edge: entire. Ventral edge: entire. Profile: plane-convex. Rostrum and antirostrum orientation: in agreement. Rostrum: underdeveloped (75\%), developed. Antirostrum: underdeveloped (75\%), developed. Pseudorostrum: underdeveloped (75\%), developed. Pseudoantirostrum: underdeveloped (75\%), developed. Sulcus acusticus: position: median; orientation: horizontal; opening: ostio-caudal; morphology: homosulcoid; colliculum: homomorphic; ostium: funnel-like; cauda: funnel-like.

The small number of otoliths examined did not permit the statistical analysis of the data but its morphometric characteristics are shown below:

\begin{tabular}{lccc}
\hline Shape indices & Mean \pm Sd & Minimum & Maximum \\
\hline OL/TL (\%) & $1.10 \pm 0.31$ & 0.70 & 1.46 \\
OH/OL (\%) & $112.93 \pm 10.68$ & 98.54 & 121.43 \\
OT/OL (\%) & $34.52 \pm 3.23$ & 32.12 & 39.29 \\
OT/OH (\%) & $30.64 \pm 2.32$ & 27.63 & 32.59 \\
Circularity & $18.51 \pm 5.61$ & 15.22 & 26.90 \\
Rectangularity & $0.69 \pm 0.05$ & 0.63 & 0.74 \\
\hline
\end{tabular}




\section{ACKNOWLEDGMENTS}

Many thanks to Thiago José Balbi for some otoliths measurements and to Alexandre Arackawa, Sílvia Gonsales, Laura Montserrat, Michelle Konig and Vanessa Sugihara for the otoliths drawings. Also special thanks to Dr. Carlos Assis for their valuable comments that improved a lot this paper. This paper received substantial financial support from the Fundação de Amparo à Pesquisa do Estado de São Paulo (FAPESP Process Numbers: 2010/51631 2 and 2014/03764 4).

\section{REFERENCES}

ALMEIDA-SILVA, P. H.; TUBINO, R. A.; ZAMBRANO, L. C.; HUNDER, D. A.; GARRITANO, S. R.; MONTEIRO-NETO, C. Trophic ecology and food consumption of fishes in a hypersaline tropical lagoon. J. Fish Biol., v. 86, n. 6, p. 1781-1795, 2015.

BERNARDES, R. A.; FIGUEIREDO, J. L.; RODRIGUES, A. R.; FISCHER, L. G.; VOOREN, C. M.; HAIMOVICI, M.; ROSSI-WONGTSCHOWSKI, C. L. D. B. Peixes da Zona Exclusiva da região Sudeste-Sul do Brasil: levantamento com armadilhas, pargueiras e rede de arrasto de fundo. São Paulo: EDUSP, 2005. p. 304.

BRENHA-NUNES, M. R.; SANTIFICETUR, C.; CONVERSANI, V. R. M.; GIARETTA, M. B.; ROSSI-WONGTSCHOWSKI, C. L. D. B.; SILIPRANDI, C. C. Atlas of marine bony fish otoliths (sagittae) of Southeastern-Southern Brazil Part IV: Perciformes (Centropomidae, Acropomatidae, Serranidae, Priacanthidae, Malacanthidae, Pomatomidae, Carangidae, Lutjanidae, Gerreidae and Haemulidae). Braz. J. Oceanogr., v. 64, n. spe 1, p. 23-75, 2016.

CARPENTER, K. E.; MUNROE, T. Atherinella brasiliensis. The IUCN Red List of Threatened Species 2015: e.T16411567A16510327, 2015. Cambridge: IUCN Global Species Programme Red List Unit, 2015.

CARVAlHO-FILHO, A. Peixes da Costa Brasileira. 2a ed. São Paulo: Marca D’Água, 1992. 304 p.

CERVIGÓN, F.; CIPRIANI, R.; FISCHER, L. W.; GARIBALDI, L.; HENDRICKX, M.; LEMUS, A. J.; MÁRQUEZ, R.; POUTIERS, J. M.; ROBAINA, G.; RODRIQUEZ, B. Fichas FAO de identificación de especies para los fines de la pesca: guía de campo de las especies comerciales marinas y de aquas salobres de la costa septentrional de Sur América. Rome: FAO, 1992. p. 513.

CHERNOFF, B. Atherinopsidae - New World silversides. In: CARPENTER, K. E.; NIEM, V. H. (Eds.). The Living Marine Resources of the Western Central Atlantic. Vol. 2. Bony fishes part 1 (Acipenseridae to Grammatidae). Rome: FAO, 2002. p. 1086-1103.

CHI-ESPÍNOLA, A. A.; VEGA-CENDEJAS, M. E. Feeding habits of Sphoeroides testudineus (Perciformes: Tetraodontidae) in the lagoon system of Ria Lagartos, Yucatán, Mexico. Rev. Biol. Trop., v. 61, n. 2, p. 849-858, 2013.

COLLETTE, B. B. Hemiramphidae. In: CARPENTER, K. E.; NIEM, V. H. (Eds.). The Living Marine Resources of the Western Central Atlantic. Vol. 2. Bony fishes part 1 (Acipenseridae to Grammatidae). Rome: FAO, 2002a. p. 1116-1144.

COLleTtE, B. B. Belonidae. In: CARPENTER, K. E.; NIEM, V. H. (Eds.). The Living Marine Resources of the Western Central Atlantic. Vol. 2. Bony fishes part 1 (Acipenseridae to Grammatidae). Rome: FAO, 2002b. p. 1104-1103.

CONTENTE, R. F.; STEFANONI, M. F.; SPACH, H. L. Feeding ecology of the Brazilian silverside Atherinella brasiliensis (Atherinopsidae) in a sub-tropical estuarine ecosystem. J. Mar. Biol. Assoc. U.K., v. 91, n. 6, p. 1197-1205, 2011.

DENADAI, M. R.; SANTOS, F. B.; BESSA, E.; BERNARDES, L. P.; TURRA, A. Population biology and diet of the puffer fish Lagocephalus laevigatus (Tetraodontiformes: Tetraodontidae) in Caraguatatuba Bay, south-eastern Brazil. J. Mar. Biol. Assoc. U.K., v. 92 , n. 2, p. 407-412, 2012.

EHRICH, S. Macroramphosidae. In: WHITEHEAD, P. J. P.; BAUCHOT, M. L.; HUREAU, J. C.; NIELSEN, J.; TORTONESE, E. (Eds.). Fishes of the North-Eastern Atlantic and the Mediterranean. Vol. 2. Paris: UNESCO, 1986. 627 p.

EHRICH, S. Macroramphosidae. In: QUERO, J. C.; HUREAU, J. C.; KARRER, C.; POST, A.; SALDANHA, L. (Eds.). Check-list of the fishes of the eastern tropical Atlantic (CLOFETA). Vol. 2. JNICT, Lisbon; SEI, Paris and UNESCO. Paris: UNESCO, 1990. p. 656-657.

FIGUEIREDO, J. L.; MENEZES, N. A. Manual de peixes marinhos do sudeste do Brasil. II. Teleostei (1). São Paulo: Museu de Zoologia da Universidade de São Paulo, 1978. 110 p.

FIGUEIREDO, J. L.; MENEZES, N. A. Manual de peixes marinhos do Sudeste do Brasil. III. Teleostei (2). São Paulo: Museu de Zoologia da Universidade de São Paulo, 1980. 90 p.

FIGUEIREDO, J. L.; MENEZES, N. A. Manual de peixes marinhos do Sudeste do Brasil. VI. Teleostei (5). São Paulo: Museu de Zoologia da Universidade de São Paulo, 2000. 116 p.

FIGUEIREDO, J. L.; SANTOS, A. P.; YAMAGUTI, N.; BERNARDES, R. A.; ROSSI-WONGTSCHOWSKI, C. L. D. B. Peixes da zona econômica exclusiva da região Sudeste-Sul do Brasil: levantamento com rede de meia-água. São Paulo: EDUSP: Imprensa Oficial do Estado de São Paulo, 2002. 242 p.

FROESE, R.; PAULY, D. (Eds.). FishBase. World Wide Web electronic publication. Available in: $<$ http://www.fishbase.org >. Version: 7. 2017. Access: 2017 Aug 16.

HEEMSTRA, P. C. Grammicolepidae. In: CARPENTER, K.E.; NIEM, V. H. (Eds.). The Living Marine Resources of the Western Central Atlantic. Vol. 2. Bony fishes part 1 (Acipenseridae to Grammatidae). Rome: FAO, 2002. p. 1214-1216.

HORN, P. L.; FORMAN, J.; DUNN, M. R. Feeding habits of alfonsino Beryx splendens. J. Fish Biol., v. 76, n. 10, p. $2382-2400,2010$.

HUIZAR, A. R.; CARRARA, X. C. Hábitos alimentarios de Diodon histrix y Diodon holocanthus (Pisces: Diodontidae), en las costas de Jalisco y Colima, México. Bol. Cen. Investig. Biol., v. 34, n. 2, p. 118-210, 2000. 
IGFA. Database of IGFA angling records until 2001. Fort Lauderdale: International Game Fish Association, 2001.

MENEZES, N. A.; BUCKUP, P. A.; FIGUEIREDO, J. L.; MOURA, R. L. (Eds.). Catálogo das espécies de peixes marinhos do Brasil. São Paulo: Museu de Zoologia da Universidade de São Paulo, 2003. 160 p.

MOORE, J. A. Berycidae. In: CARPENTER, K. E.; NIEM, V. H. (Eds.). The Living Marine Resources of the Western Central Atlantic. Vol. 2. Bony fishes part 1 (Acipenseridae to Grammatidae). Rome: FAO, 2002a. p. 1189-1191.

MOORE, J. A. Trachichthyidae. In: CARPENTER, K. E.; NIEM, V. H. (Eds.). FAO species identification guide for fishery purposes. The Living Marine Resources of the Western Central Atlantic. Vol. 2. Bony fishes part 1 (Acipenseridae to Grammatidae). Rome: FAO, 2002b. p. 1184-1188.

PAXTON, J. R. Berycidae. Alfonsinos. In: CARPENTER, K. E.; NIEM, V. H. (Eds.). The Living Marine Resources of the Western Central Atlantic. Vol. 4. Bony fishes part 2 (Mugilidae to Carangidae). Rome: FAO, 1999. p. 2218-2224.

RICHARDS, W. J. Triglidae. In: CARPENTER, K. E.; NIEM, V. H. (Eds.). The Living Marine Resources of the Western Central Atlantic. Vol. 2. Bony fishes part 1 (Acipenseridae to Grammatidae). Rome: FAO, 2002. p. 1245-1285.

ROSSI-WONGTSCHOWSKI, C. L. D. B.; SILIPRANDI, C. C.; BRENHA, M. R.; GONSALES, S. A.; SANTIFICETUR, C.; VAZ-DOS-SANTOS, A. M. Atlas of marine bony fish otoliths (Sagittae) of Southeastern - Southern Brazil Part I: Gadiformes (Macrouridae, Moridae, Bregmacerotidae, Phycidae and Merlucciidae); Part II: Perciformes (Carangidae, Sciaenidae, Scombridae and Serranidae). Braz. J. Oceanogr., v. 62, n. spe 1, p. 1-103, 2014

ROSSI-WONGTSCHOWSKI, C. L. D. B.; CHALOM, A.; SILIPRANDI, C. C.; BRENHA-NUNES, M. R.; CONVERSANI, V. R. M.; SANTIFICETUR, C.; GIARETTA, M.B. 2016. COSS-Brasil: Coleção de Otólitos de Peixes Marinhos da Região Sudeste-Sul do Brasil. Instituto Oceanográfico da Universidade de São Paulo. www.usp.br/cossbrasil (versão 2016).

SÃO CLEMENTE, R. R. B.; COSTA, P. A. S.; MARTINS, A. S. Distribution and feeding habits of three sea robin species (Bellator brachychir, Prionotus nudigula and Prionotus punctatus) in the Campos Basin, Southeastern Brazil. Lat. Am. J. Aquat. Res., v. 42, n. 3 , p. 488-496, 2014.

SCHNEIDER, W. FAO species identification sheets for fishery purposes. Field guide to the commercial marine resources of the Gulf of Guinea. Prepared and published with the support of the FAO Regional Office for Africa. Rome: FAO, 1990. $268 \mathrm{p}$.

SILIPRANDI, C. C.; BRENHA-NUNES, M. R.; ROSSI-WONGTSCHOWSKI, C. L. D. B.; SANTIFICETUR, C.; CONVERSANI, V. R. M. Atlas of marine bony fish otoliths (sagittae) of Southeastern-Southern Brazil Part III: Clupeiformes (Clupeidae, Engraulidae, Pristigasteridae). Braz. J. Oceanogr., 64, n. spe 1, p. 1-22, 2016.

SOARES, L. S. H.; APELBAUM, R. Atividade alimentar diária da cabrinha Prionotus punctatus (Teleostei: Triglidae) do litoral de Ubatuba, Brasil. Bol. Inst. Oceanogr., v. 42, n. 1/2, p. 85-98, 1994.

SOMMER, C.; SCHNEIDER, W.; POUTIERS, J. M. FAO species identification field guide for fishery purposes. The Living Marine Resources of Somalia. Rome: FAO, 1996. 376 p. 
A1

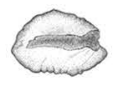

A2

A3

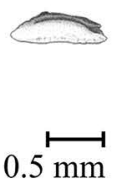

A1

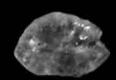

B2

B3

B1

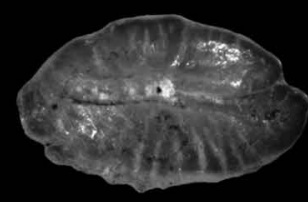

A2

B2

A3

B3

thes

$0.5 \mathrm{~mm}$
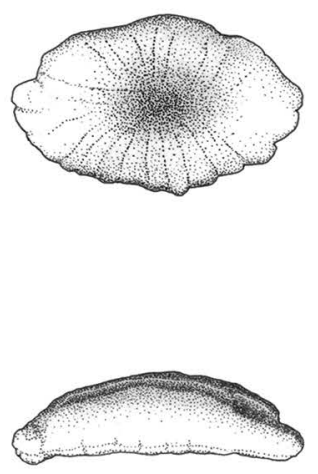

$\longmapsto 1 \mathrm{~mm}$

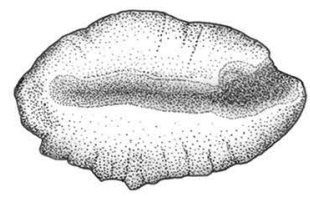

C1

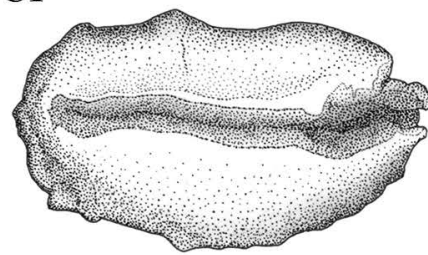

C2

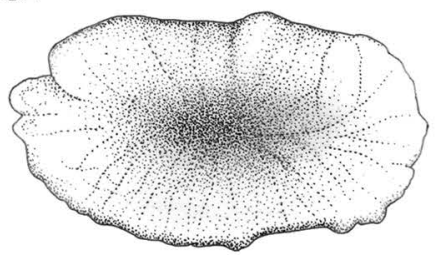

C3

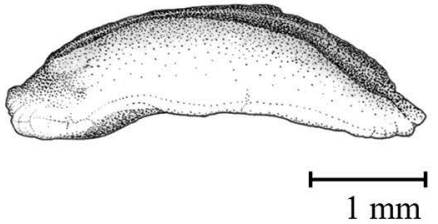

\section{C1}

C2

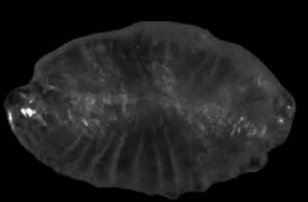

C3

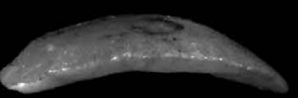

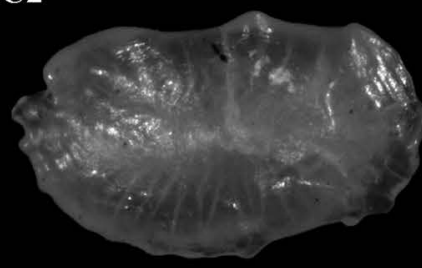
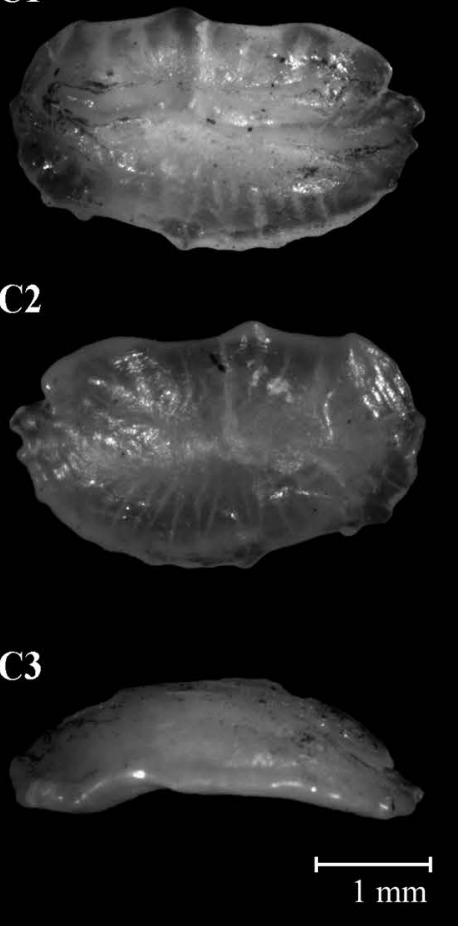

Plate 1. Illustrations (above) and photographs (below) of Atherinella brasiliensis otoliths from fish with total length: A. $32 \mathrm{~mm}$; B. $86 \mathrm{~mm}$; C. $141 \mathrm{~mm}$. The medial face is shown in A1; B1; C1; the lateral face in A2; B2; C2; and the ventral profile in A3; B3, C3 (Illustration: Laura Montserrat; Photos: Cesar Santificetur). 
A1

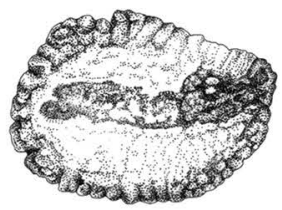

A2

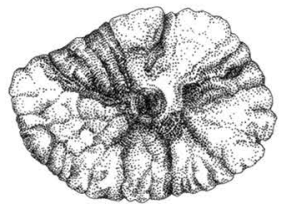

A3

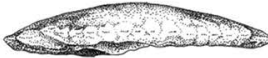

$1 \mathrm{~mm}$
B1

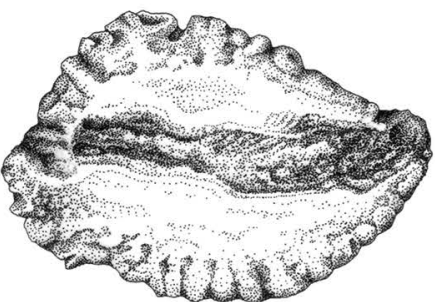

B2

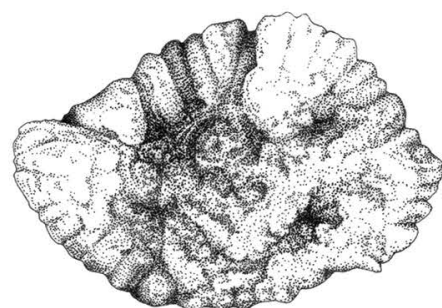

B3

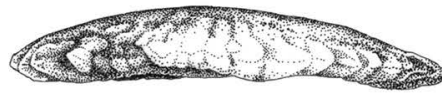

$1 \mathrm{~mm}$
A1

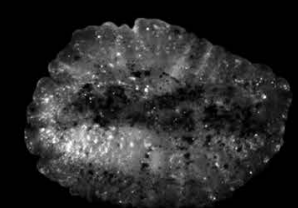

A2

A3

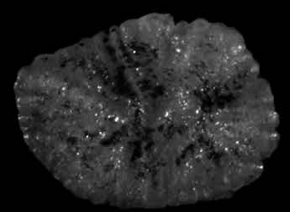

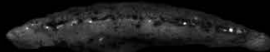

B1

B2

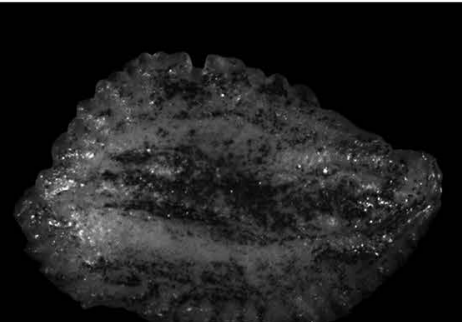

B3

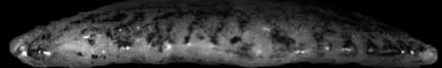

Plate 2. Illustrations (above) and photographs (below) of Hemiramphus brasiliensis otoliths from fish with total length: A. $116 \mathrm{~mm}$; B. $200 \mathrm{~mm}$. The medial face is shown in A1; B1; the lateral face in A2; B2; and the ventral profile in A3; B3 (Illustration: Sílvia de Almeida Gonsales; Photos: Cesar Santificetur). 

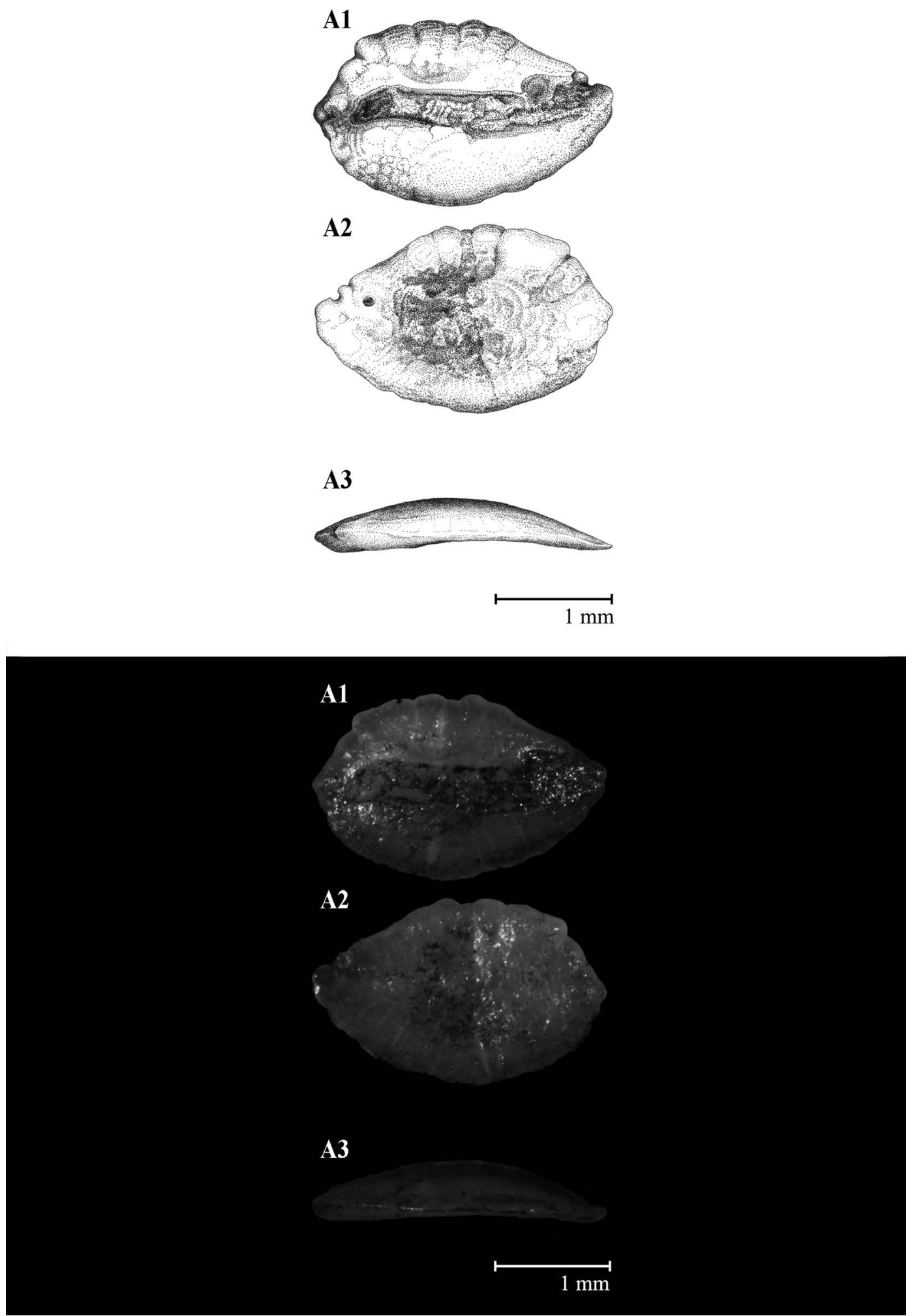

Plate 3. Illustrations (above) and photographs (below) of Hyporhamphus roberti otolith from fish with total length: A. $132 \mathrm{~mm}$. The medial face is shown in A1; the lateral face in A2; and the ventral profile in A3 (Illustration: Sílvia de Almeida Gonsales; Photos: Cesar Santificetur). 
A1

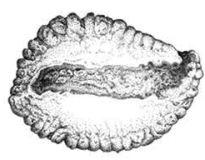

A2

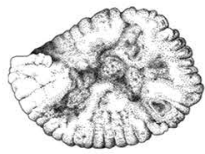

A3

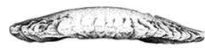

$1 \mathrm{~mm}$
B1

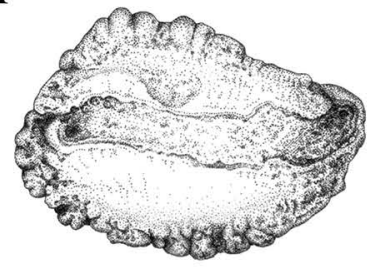

B2

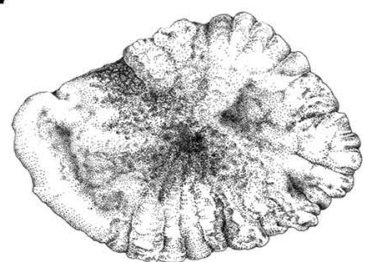

B3

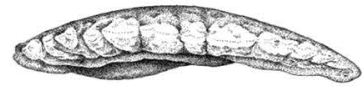

$\stackrel{\longmapsto}{1 \mathrm{~mm}}$
C1

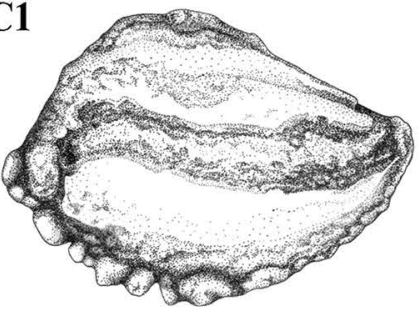

C2

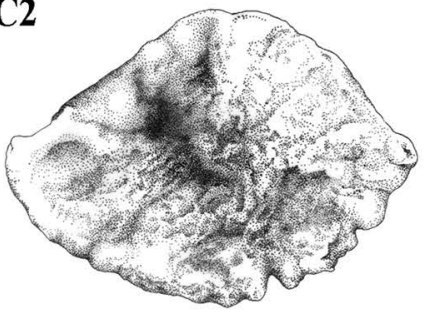

C3

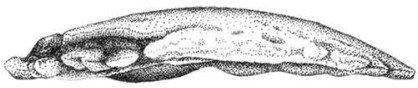

A1

A2
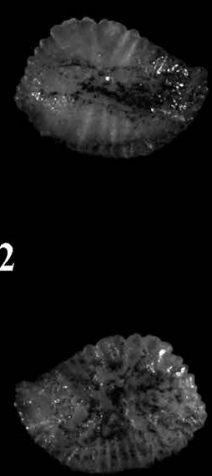

A3

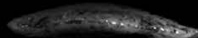

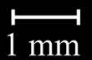

$1 \mathrm{~mm}$
B1

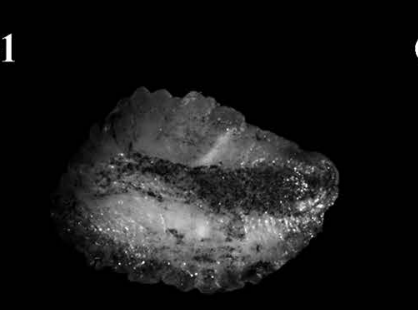

B2

B3

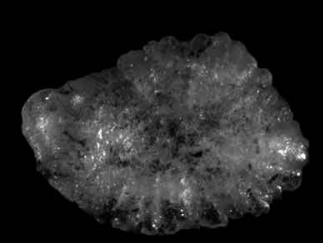

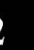

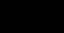

33

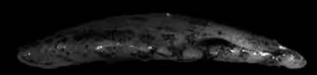

C1

C2

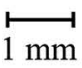

C3

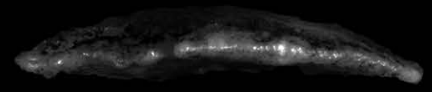

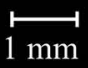

Plate 4. Illustrations (above) and photographs (below) of Hyporhamphus unifasciatus otoliths from fish with total length: A. $116 \mathrm{~mm}$; B. $190 \mathrm{~mm}$; C. $261 \mathrm{~mm}$. The medial face is shown in A1; B1; C1; the lateral face in A2; B2; C2; and the ventral profile in A3; B3; C3 (Illustration: Sílvia de Almeida Gonsales; Photos: Cesar Santificetur). 
A1

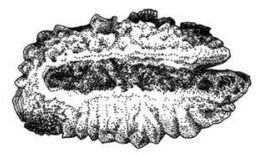

A2

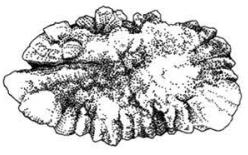

A3

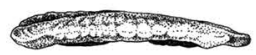

B1

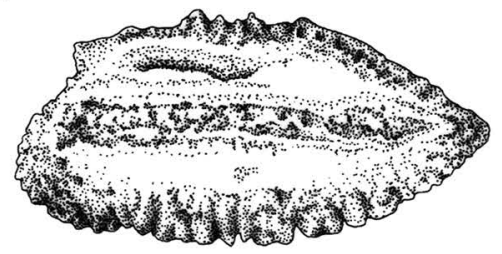

B2

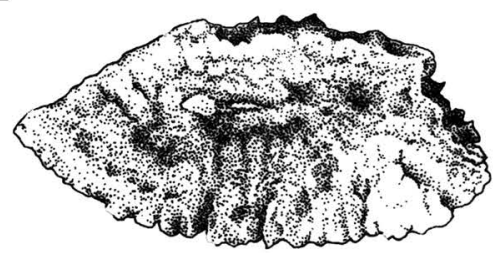

B3

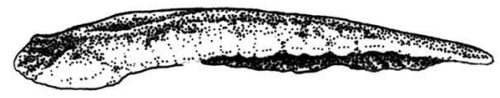

$\stackrel{\longmapsto}{1 \mathrm{~mm}}$

A1

B1
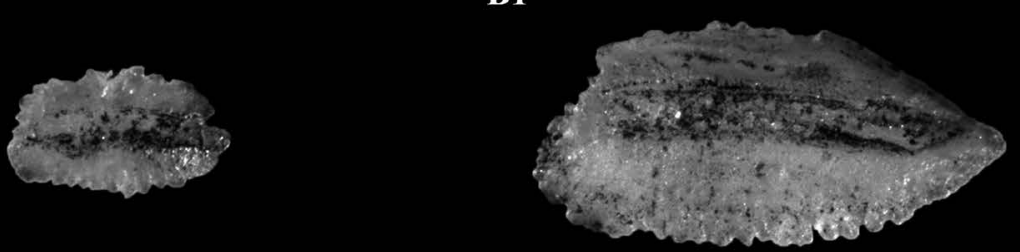

A2

B2
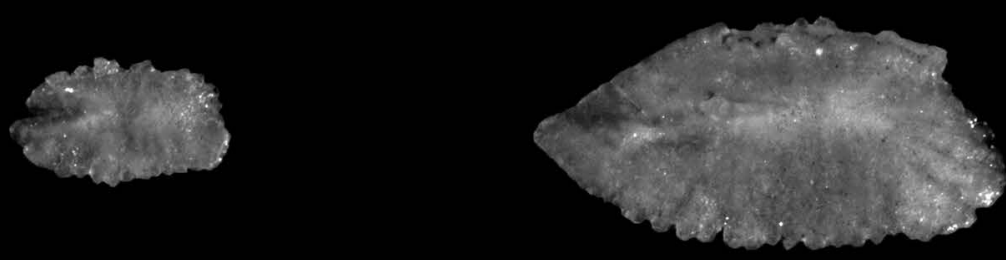

A3

B3
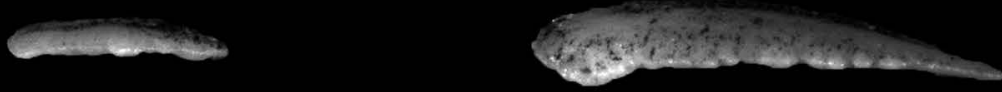

$$
\stackrel{\longmapsto \mathrm{mm}}{\longmapsto}
$$

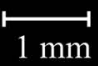

Plate 5. Illustrations (above) and photographs (below) of Strongylura marina otoliths from fish with total length: A. $283 \mathrm{~mm}$; B. $621 \mathrm{~mm}$. The medial face is shown in A1; B1; the lateral face in A2; B2; and the ventral profile in A3; B3 (Illustration: Michelle Konig; Photos: Cesar Santificetur). 

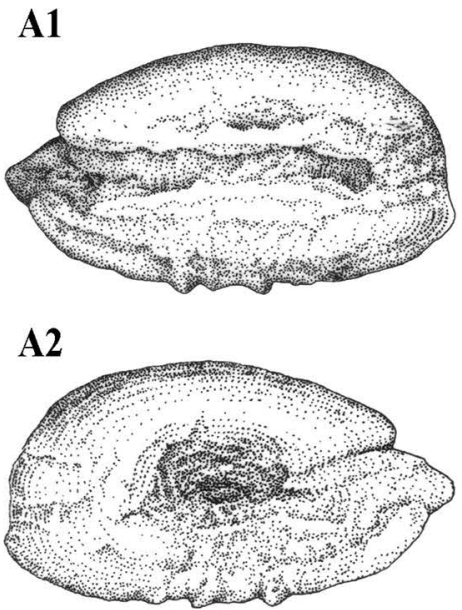

A3
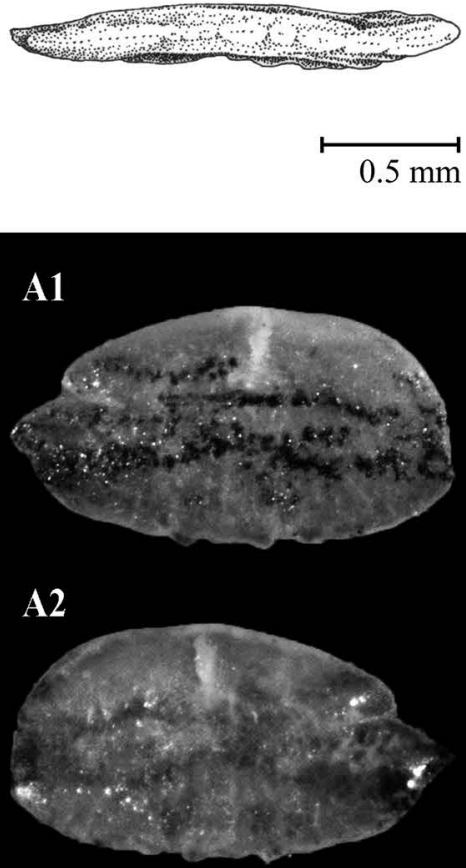

A3
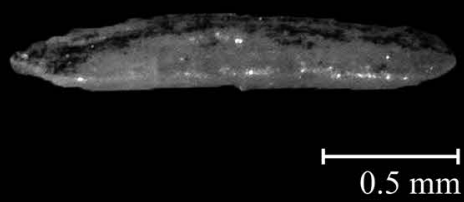

Plate 6. Illustrations (above) and photographs (below) of Strongylura timucu right otolith from fish with total length: A. $156 \mathrm{~mm}$. The medial face is shown in A1; the lateral face in A2; and the ventral profile in A3 (Illustration: Michelle Konig; Photos: Cesar Santificetur). 
A1

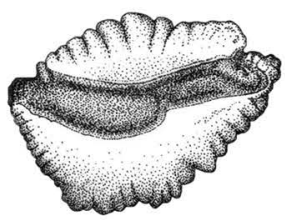

A2

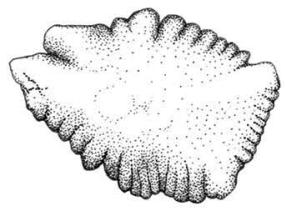

A3

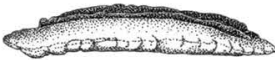

$2 \mathrm{~mm}$
B1

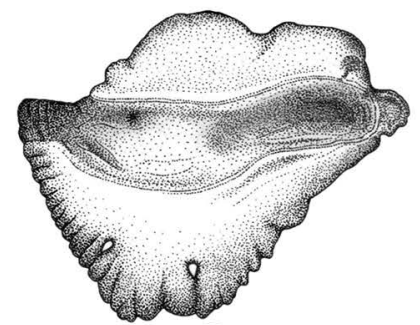

B2

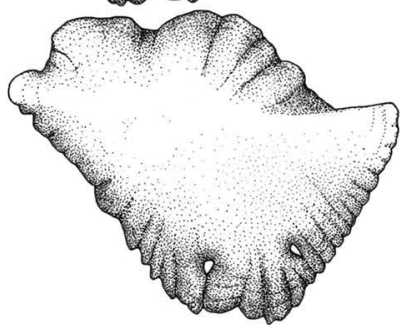

B3

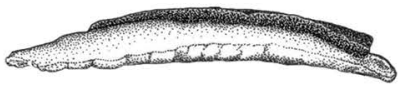

$\stackrel{\longmapsto}{2 \mathrm{~mm}}$
A1

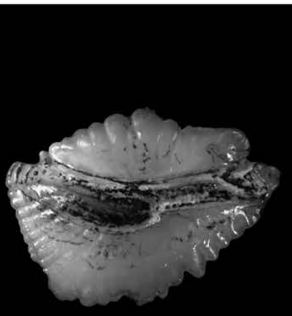

A2

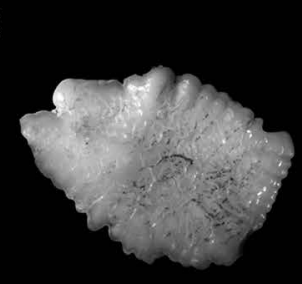

A3
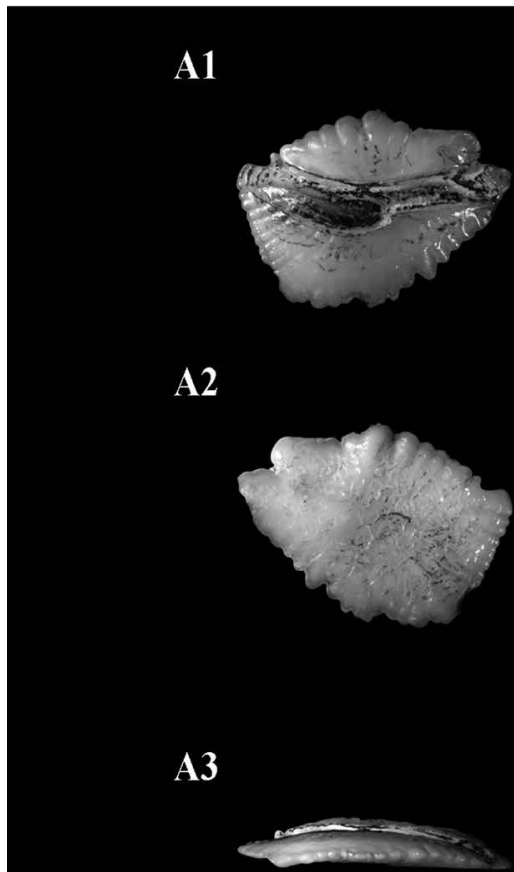

.

3

(1)
B1

B2
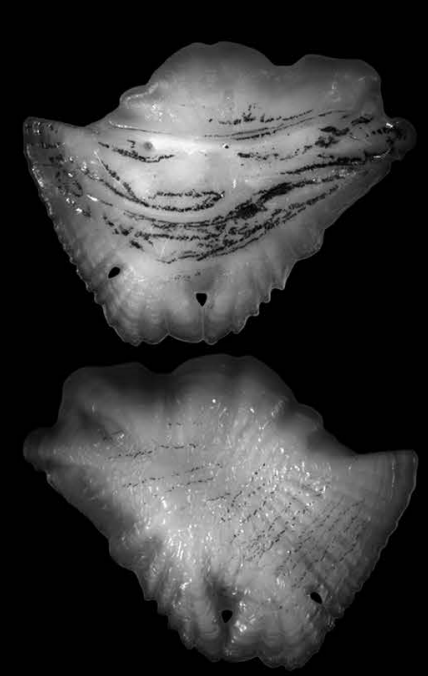

B3

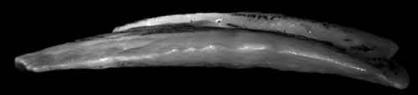

\section{$2 \mathrm{~mm}$}

Plate 7. Illustrations (above) and photographs (below) of Beryx splendens right otoliths from fish with total length: A. $170 \mathrm{~mm}$; B. $314 \mathrm{~mm}$. The medial face is shown in A1; B1; the lateral face in A2; B2; and the ventral profile in A3; B3 (Illustration: Laura Montserrat; Photos: Cesar Santificetur). 


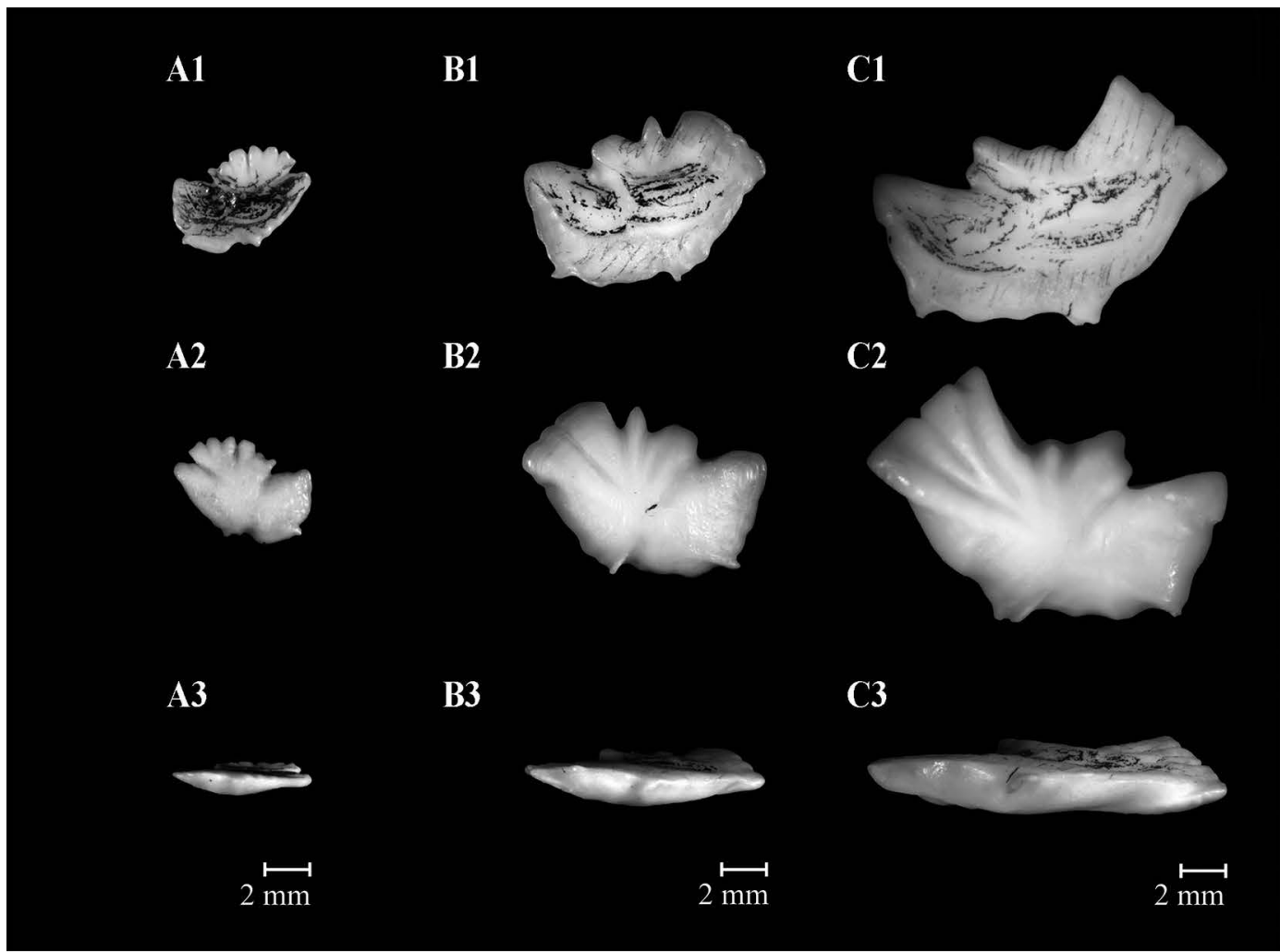

A1

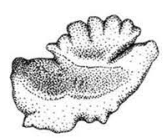

A2

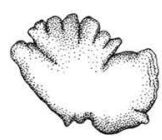

A3

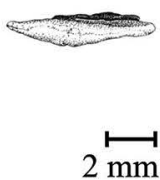

B1

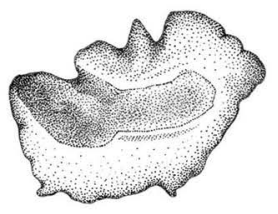

B2

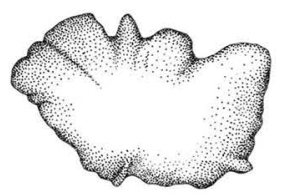

B3

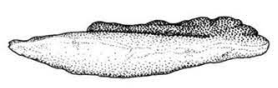

$2 \stackrel{\longmapsto}{\mathrm{mm}}$
C1

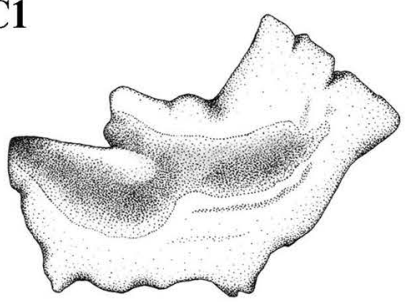

C2

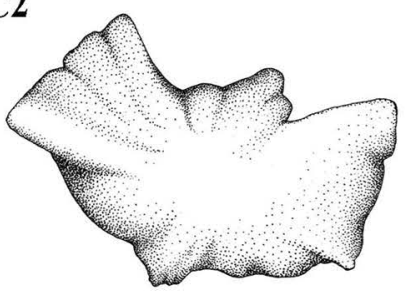

C3

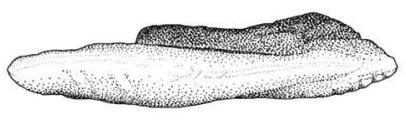

$2 \stackrel{\mathrm{mm}}{\longmapsto}$

Plate 8. Illustrations (above) and photographs (below) of Hoplostethus occidentalis right otoliths from fish with total length: A. 93 mm; B. 169 $\mathrm{mm}$; C. $245 \mathrm{~mm}$. The medial face is shown in A1; B1; C1; the lateral face in A2; B2; C2; and the ventral profile in A3; B3; C3 (Illustration: Laura Montserrat; Photos: Cesar Santificetur). 
A1

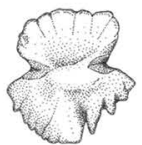

A2

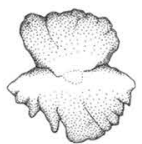

A3

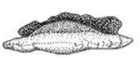

$2 \mathrm{~mm}$
B1

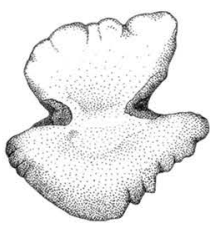

B2

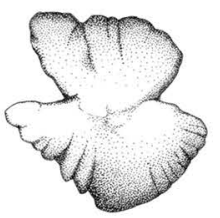

B3

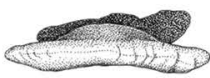

$2 \mathrm{~mm}$
C1

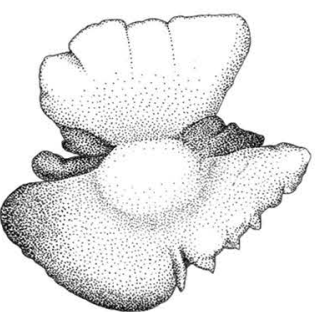

C2

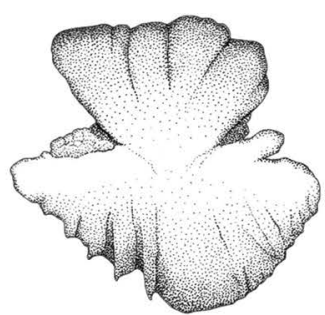

C3

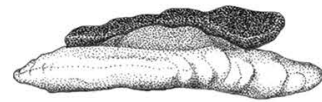

$2 \mathrm{~mm}$
A1

B1

B2

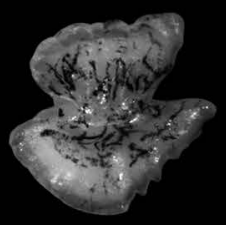

A2
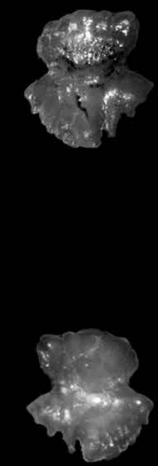

A3

$2+\infty+\infty$

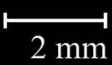

B3
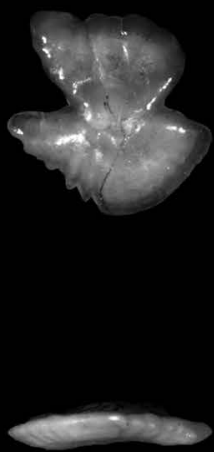

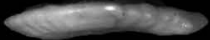

C2

C1
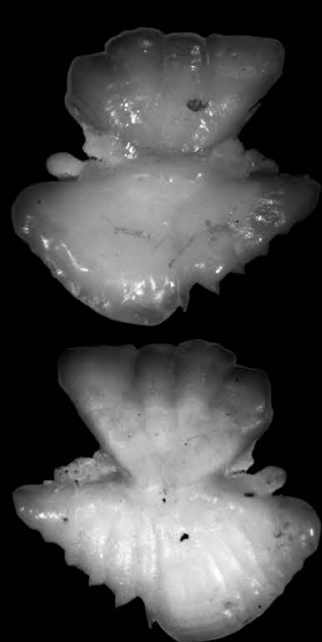

C3

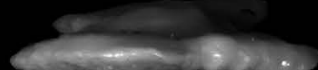

Plate 9. Illustrations (above) and photographs (below) of Xenolepidichthys dalgleishi otoliths from fish with total length: A. 68 mm; B. 127 mm; C. $260 \mathrm{~mm}$. The medial face is shown in A1; B1; C1; the lateral face in A2; B2; C2; and the ventral profile in A3; B3; C3 (Illustration: Laura Montserrat; Photos: Cesar Santificetur). 
A1

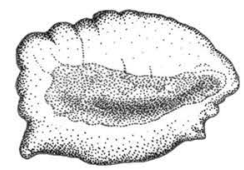

A2

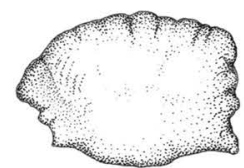

A3

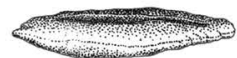

$\stackrel{\mathrm{mm}}{\longmapsto}$
B1

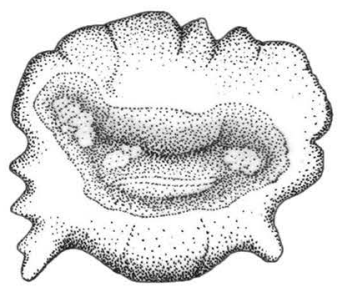

B2

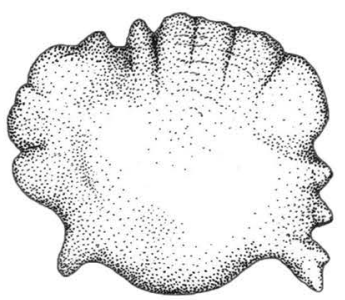

B3

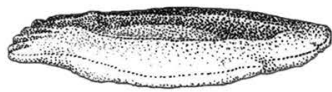

$\stackrel{\longmapsto}{1 \mathrm{~mm}}$
C1

C2
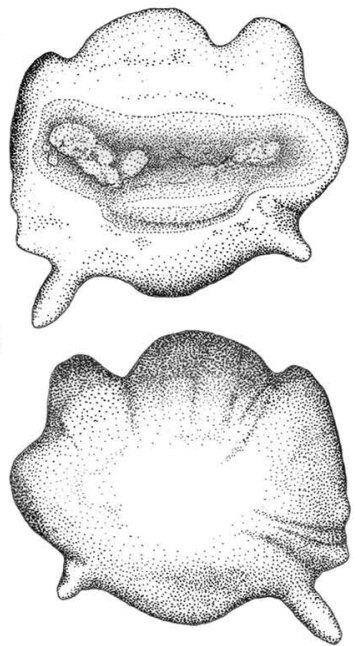

C3

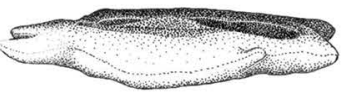

$1 \mathrm{~mm}$

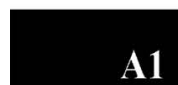

A1

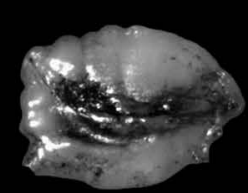

A2

A3

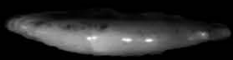

$1 \mathrm{~mm}$
B1

B2

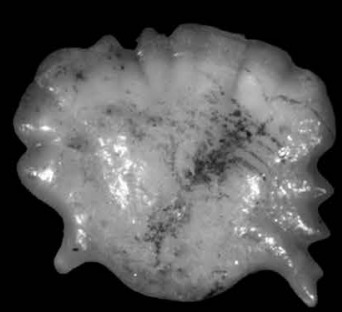

B3

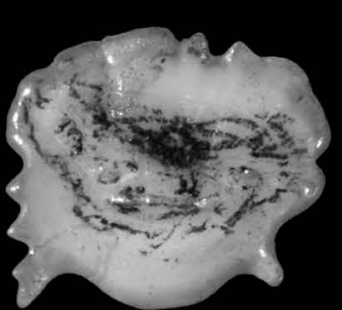

$\mathrm{C} 2$

C1

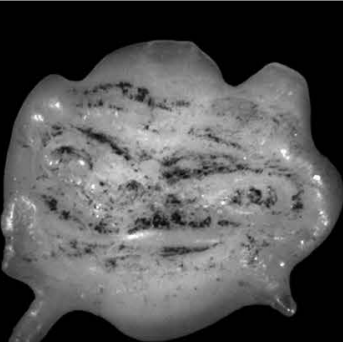

2

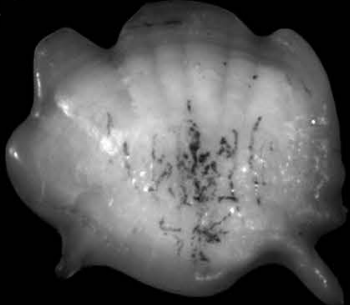

C3
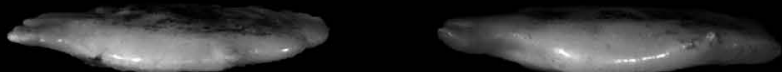

2)

\section{$1 \mathrm{~mm}$}

Plate 10. Illustrations (above) and photographs (below) of Zenion hololepis otoliths from fish with total length: A. $56 \mathrm{~mm}$; B. $114 \mathrm{~mm}$; C. 173 $\mathrm{mm}$. The medial face is shown in $\mathrm{A} 1$; $\mathrm{B} 1 ; \mathrm{C} 1$; the lateral face in $\mathrm{A} 2$; $\mathrm{B} 2$; $\mathrm{C} 2$; and the ventral profile in A3; $\mathrm{B} 3$; $\mathrm{C} 3$ (Illustration: Laura Montserrat; Photos: Cesar Santificetur). 

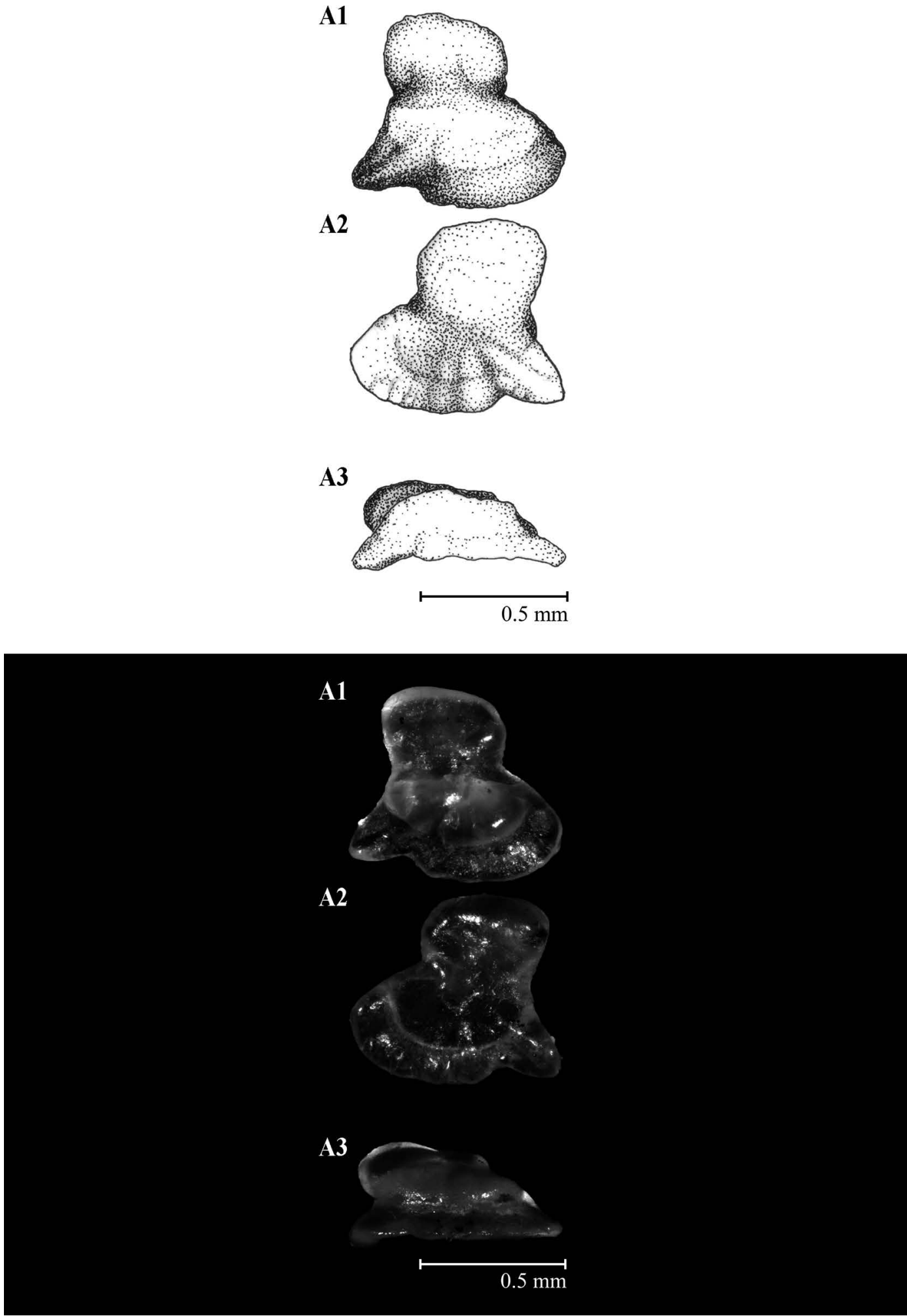

Plate 11. Illustrations (above) and photographs (below) of Macroramphosus scolopax otolith from fish with total length: A. $107 \mathrm{~mm}$. The medial face is shown in A1; the lateral face in A2; and the ventral profile in A3 (Illustration: Alexandre Arackawa; Photos: Cesar Santificetur). 
A1

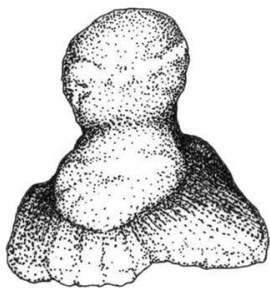

A2

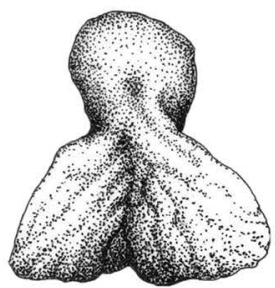

A3

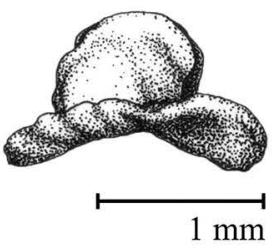

A1

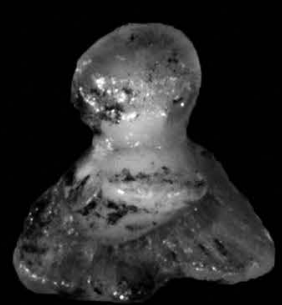

A2

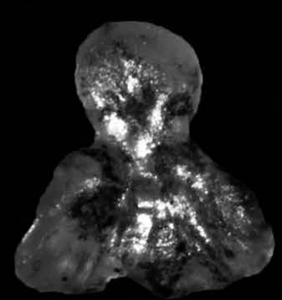

A3

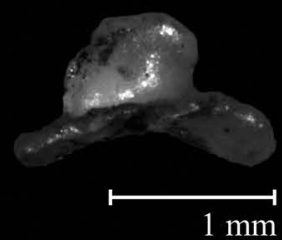

Plate 12. Illustrations (above) and photographs (below) of Notopogon fernandezianus otolith from fish with total length: A. $161 \mathrm{~mm}$. The medial face is shown in A1; the lateral face in A2; and the ventral profile in A3 (Illustration: Alexandre Arackawa; Photos: Cesar Santificetur). 
A1

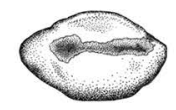

A2

A3
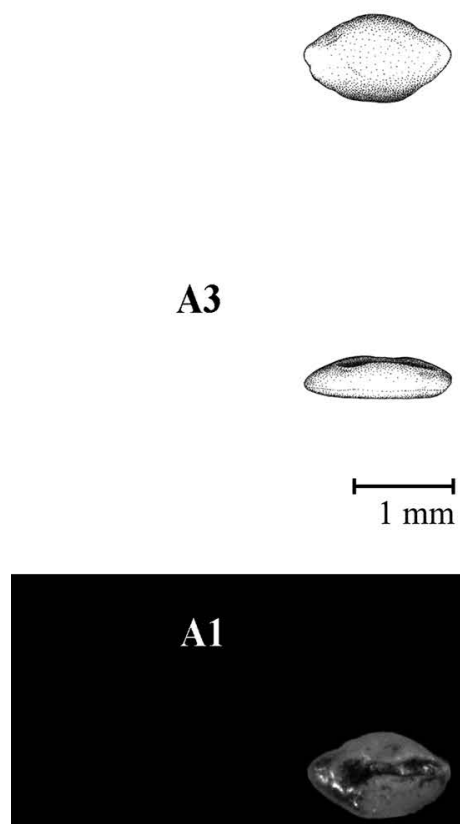

B1

A2

B2

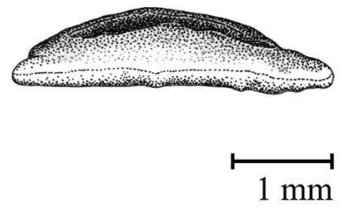

B3

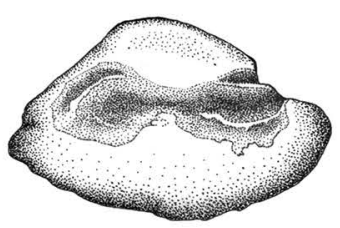

B2

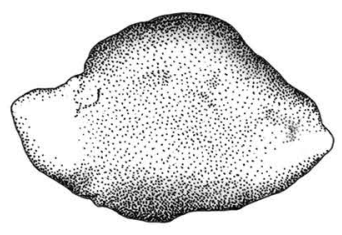

$1 \mathrm{~mm}$
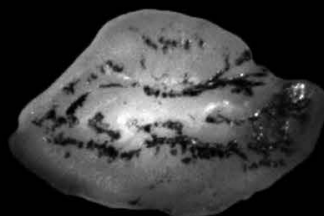

i

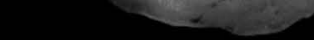

2
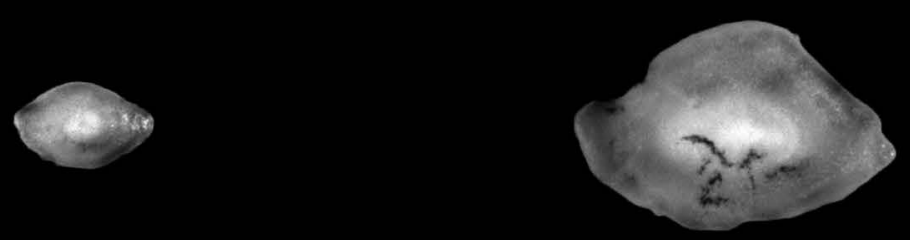

A3

B3
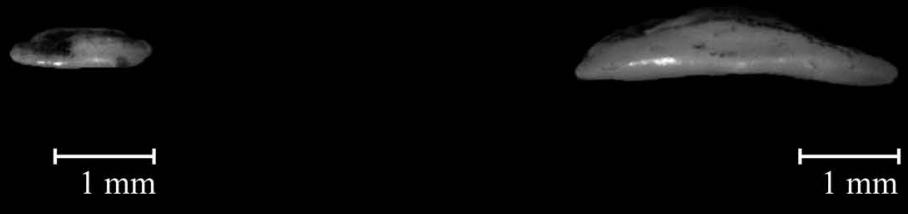

Plate 13. Illustrations (above) and photographs (below) of Bellator brachychir otoliths from fish with total length: A. $34 \mathrm{~mm}$; B. $95 \mathrm{~mm}$. The medial face is shown in A1; B1; the lateral face in A2; B2; and the ventral profile in A3; B3 (Illustration: Laura Montserrat; Photos: Cesar Santificetur). 
A1

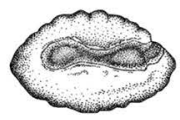

A2

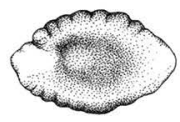

A3

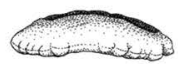

$\stackrel{\longmapsto}{1 \mathrm{~mm}}$
B1

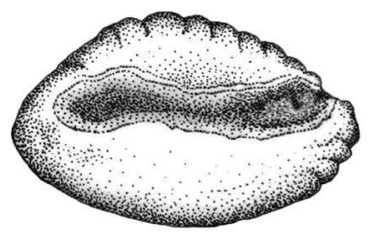

B2

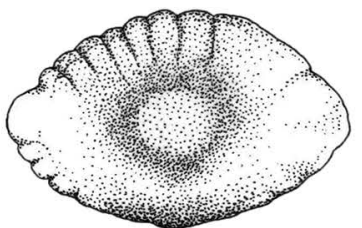

B3

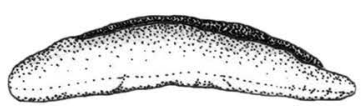

$\longmapsto$
C1

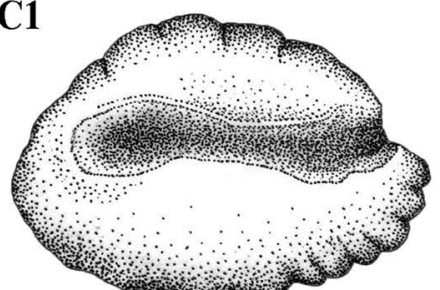

C2

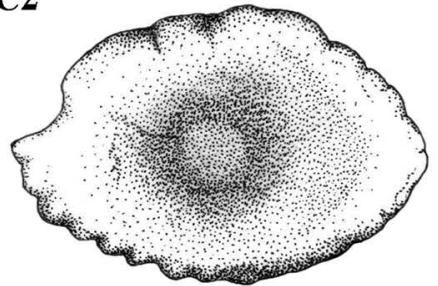

C3
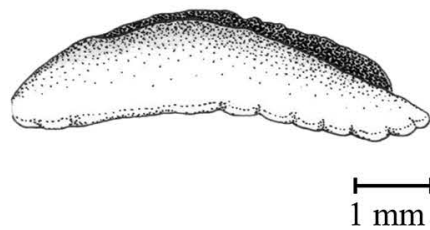

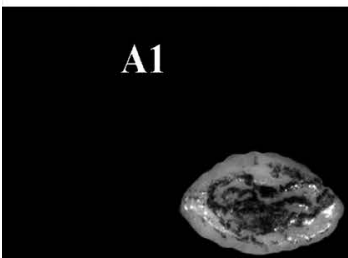

B1

C1

A2

B2
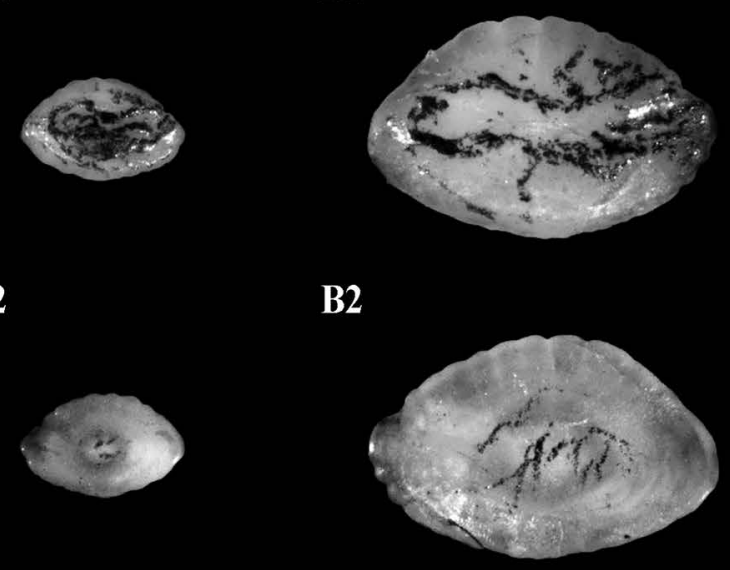

C2

A3

B3

C3
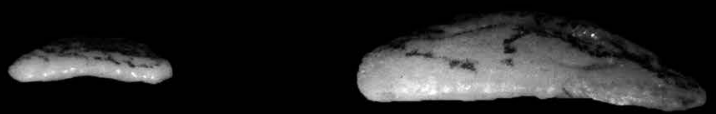

$\longmapsto$
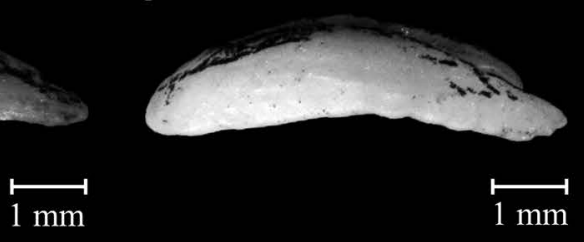

Plate 14. Illustrations (above) and photographs (below) of Prionotus nudigula otoliths from fish with total lengths: A. $68 \mathrm{~mm}$; B. $162 \mathrm{~mm}$; C. 265 $\mathrm{mm}$. The medial face is shown in A1, B1, C1; the lateral face in A2, B2, C2; and the ventral profile in A3, B3, C3 (Illustration: Laura Montserrat; Photos: Cesar Santificetur). 
A1

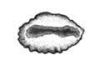

A2

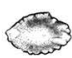

A3

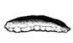

$\longmapsto$
B1

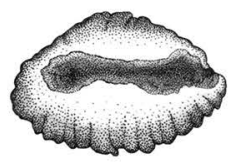

B2

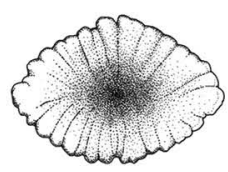

B3

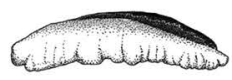

$1 \mathrm{~mm}$

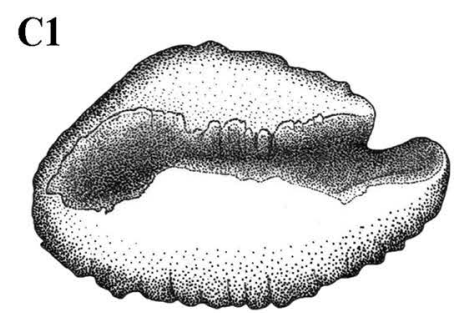

C2

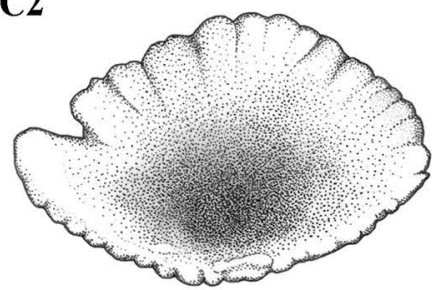

C3

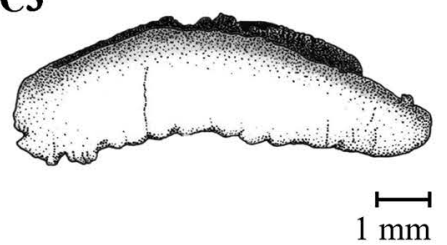

A1

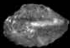

A2

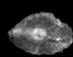

A3

$1 \mathrm{~mm}$
B1

B2

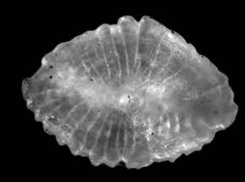

B3

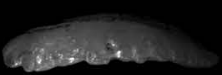

\section{C1}

C2

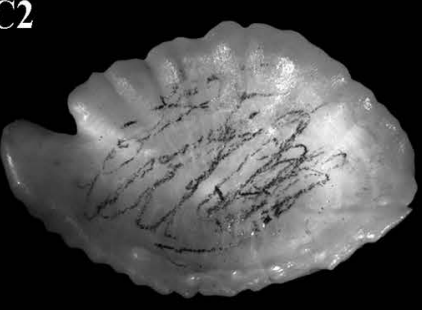

C3

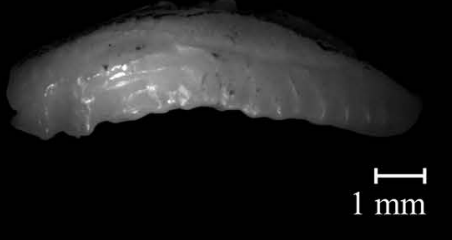

Plate 15. Illustrations (above) and photographs (below) of Prionotus punctatus otoliths from fish with total lengths: A. $36 \mathrm{~mm}$; B. $184 \mathrm{~mm}$; C. 391 $\mathrm{mm}$. The medial face is shown in A1, B1, C1; the lateral face in A2, B2, C2; and the ventral profile in A3, B3, C3 (Illustration: Laura Montserrat; Photos: Cesar Santificetur). 
A1

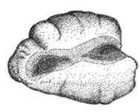

A2

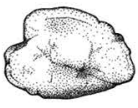

A3

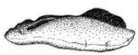

$\longmapsto$
B1

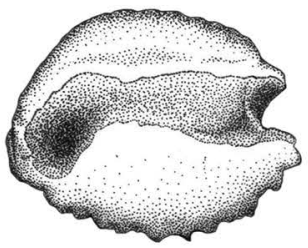

B2

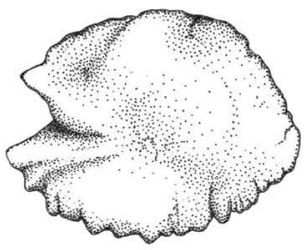

B3

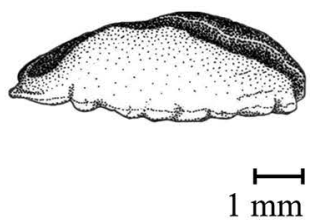

C1

\section{C2}
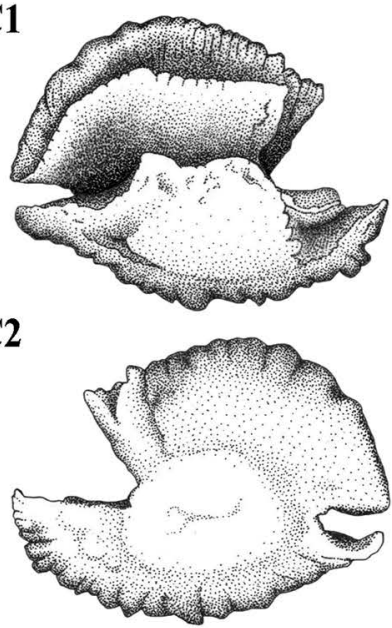

C3

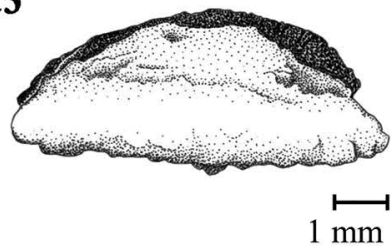

A1

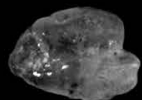

A2

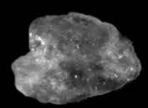

A3

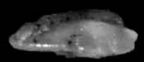

B1

B2
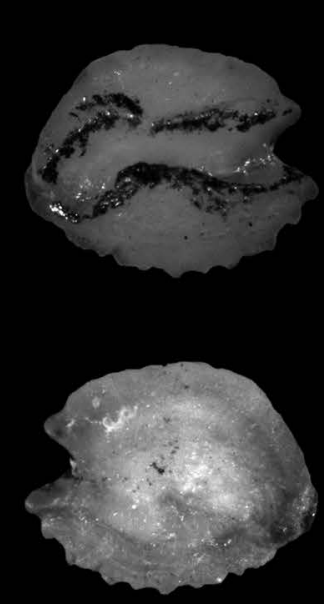

C1

C2

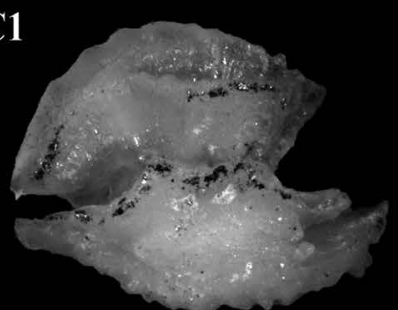

C3

B3

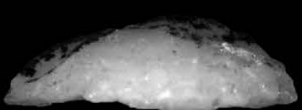

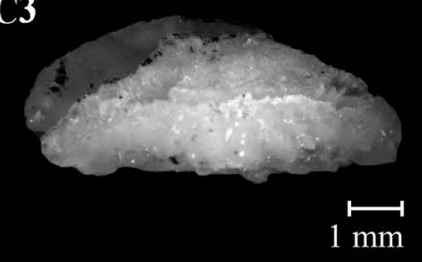

Plate 16. Illustrations (above) and photographs (below) of Dactylopterus volitans otoliths from fish with total lengths: A. $65 \mathrm{~mm}$; B. $192 \mathrm{~mm}$; C. $272 \mathrm{~mm}$. The medial face is shown in A1, B1, C1; the lateral face in A2, B2, C2; and the ventral profile in A3, B3, C3 (Illustration: Laura Montserrat; Photos: Cesar Santificetur). 
A1

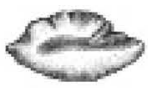

A2

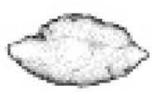

A3

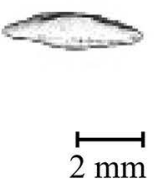

B1

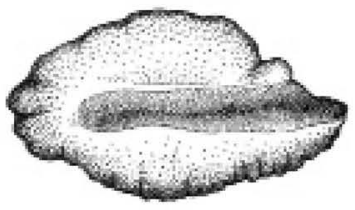

B2

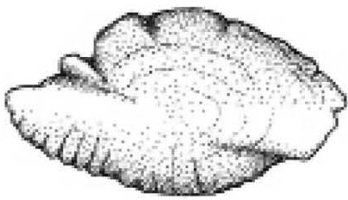

B3

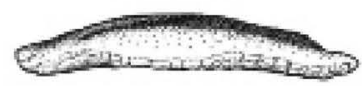

$\stackrel{\longmapsto}{2 \mathrm{~mm}}$

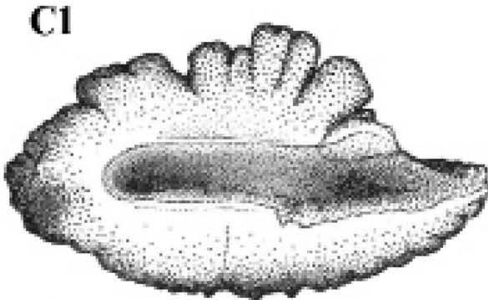

C2

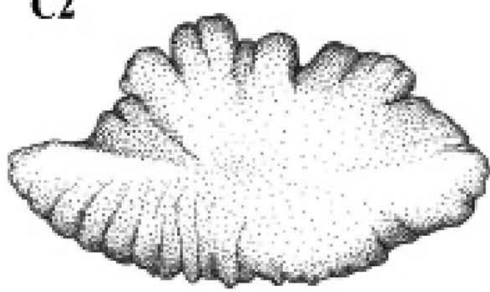

C3

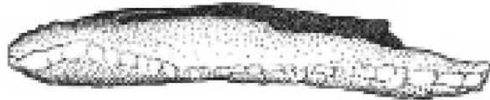

$2 \mathrm{~mm}$

A1

B1

\section{C1}
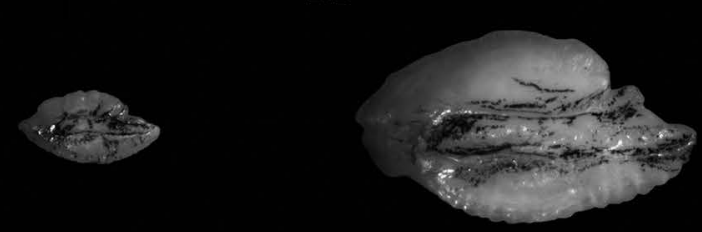

A2

B2

$\mathrm{C} 2$
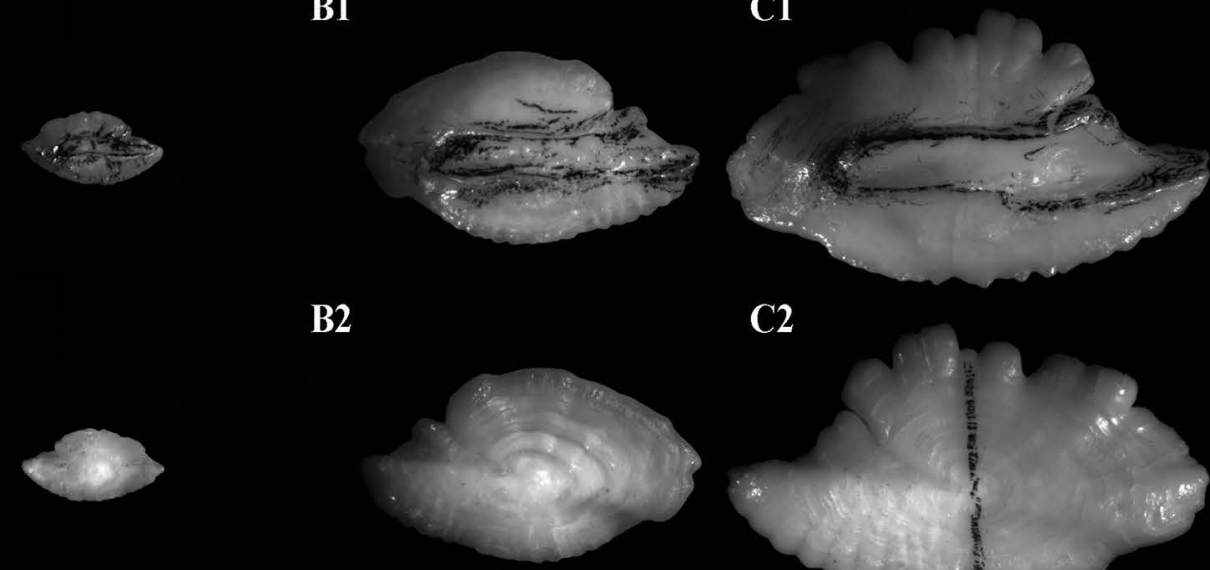

(1)

A3

B3

C3

20
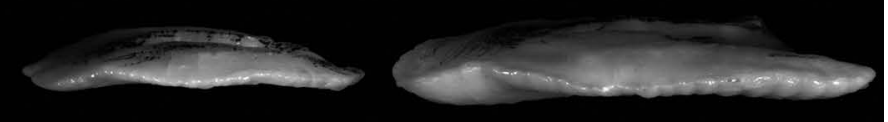

$\stackrel{\longmapsto \mathrm{mm}}{\longmapsto}$
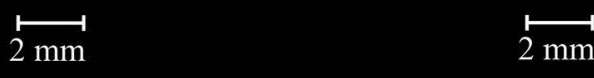

Plate 17. Illustrations (above) and photographs (below) of Helicolenus lahillei otoliths from fish with total lengths: A. $75 \mathrm{~mm}$; B. $260 \mathrm{~mm}$; C. $462 \mathrm{~mm}$. The medial face is shown in A1, B1, C1; the lateral face in A2, B2, C2; and the ventral profile in A3, B3, C3 (Illustration: Laura Montserrat; Photos: Cesar Santificetur). 
A1

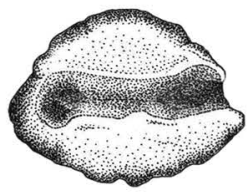

A2

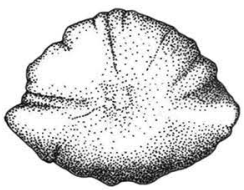

A3

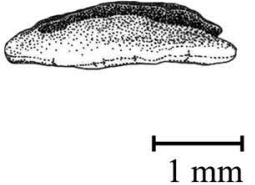

B1

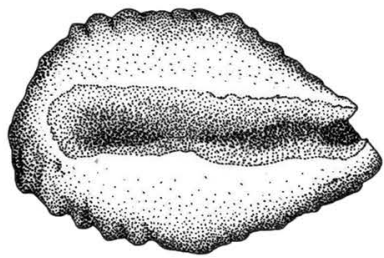

B2

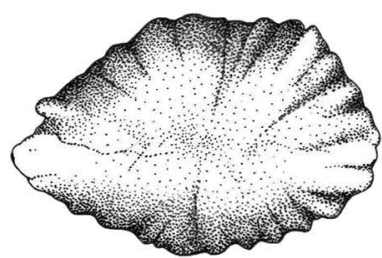

B3

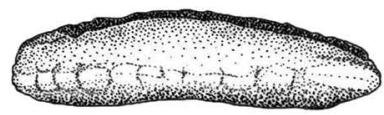

$\stackrel{\longmapsto}{1 \mathrm{~mm}}$

A1

B1

A2

B2
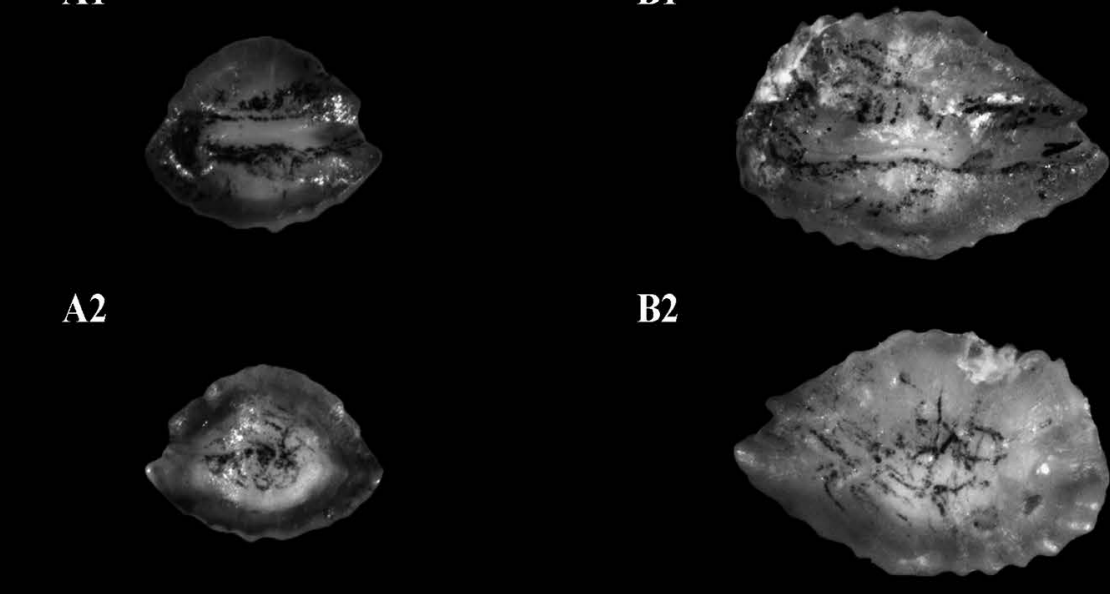

A2

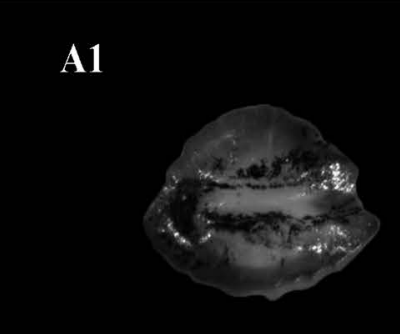

B1

A3

B3
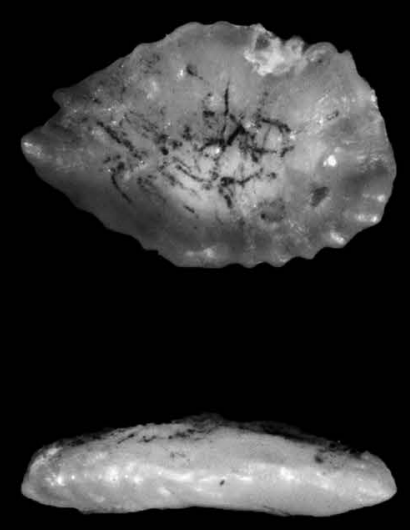

Plate 18. Illustrations (above) and photographs (below) of Peristedion altipinne otolith from fish with total lengths: A. $124 \mathrm{~mm}$; B. $258 \mathrm{~mm}$. The medial face is shown in A1, B1; the lateral face in A2, B2; and the ventral profile in A3, B3 (Illustration: Laura Montserrat; Photos: Cesar Santificetur). 

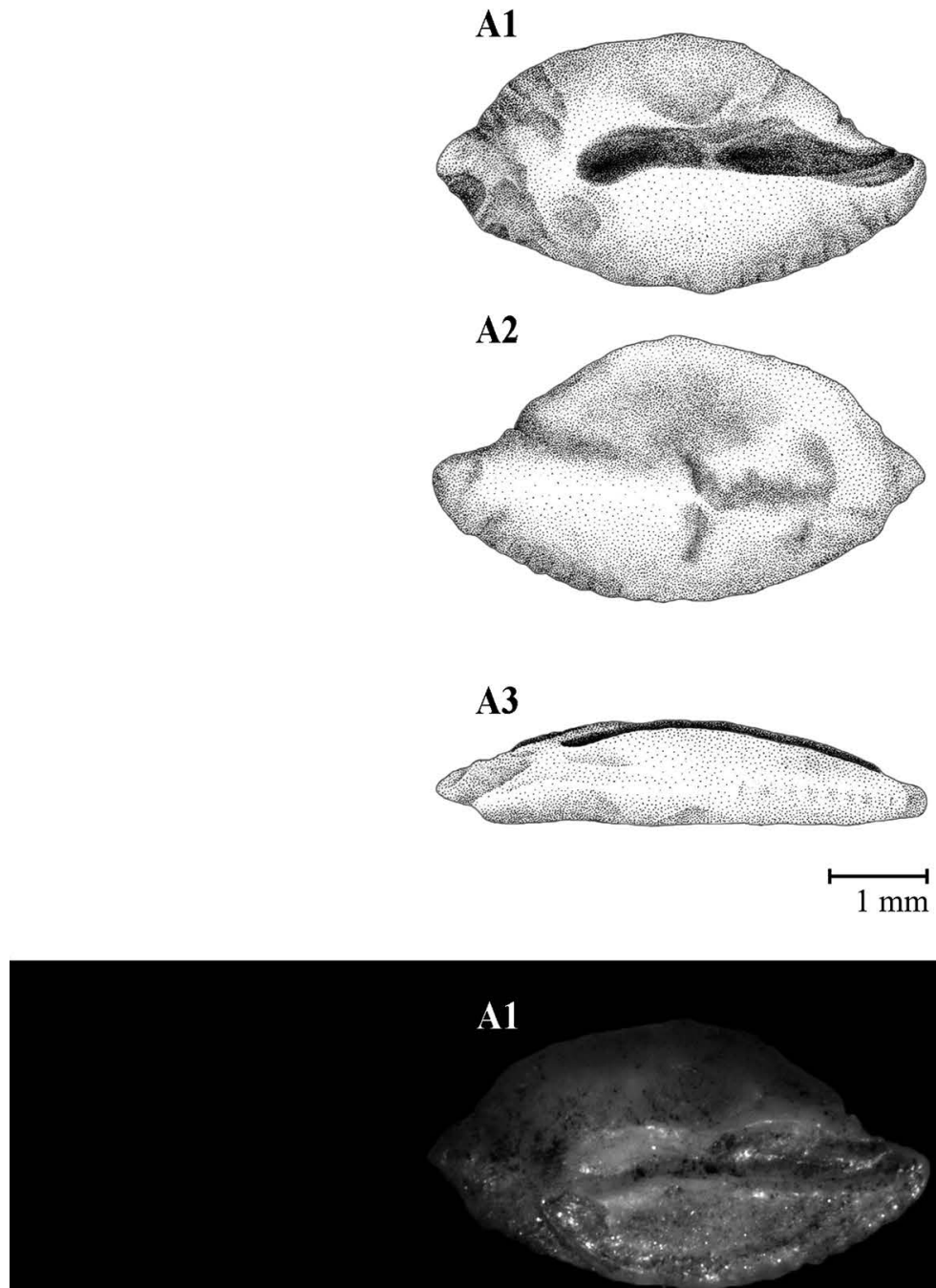

A2

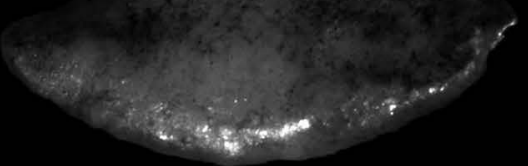

A3

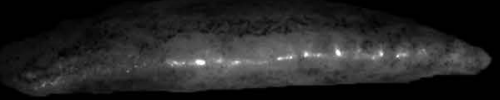

$1 \mathrm{~mm}$

Plate 19. Illustrations (above) and photographs (below) of Pontinus rathbuni otolith from fish with total length: A. $97 \mathrm{~mm}$. The medial face is shown in A1; the lateral face in A2; and the ventral profile in A3 (Illustration: Vanessa Sugihara; Photos: Cesar Santificetur). 
A1

A2

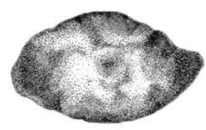

A3

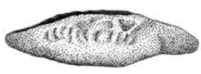

$\stackrel{\mathrm{mm}}{\longrightarrow}$
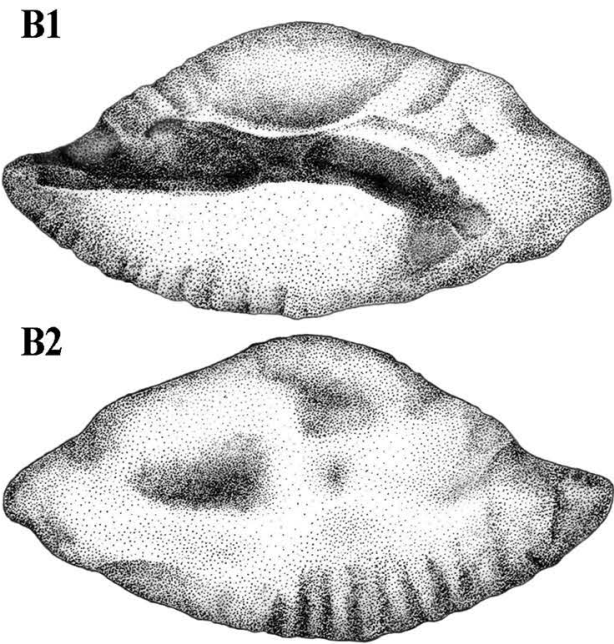

B3
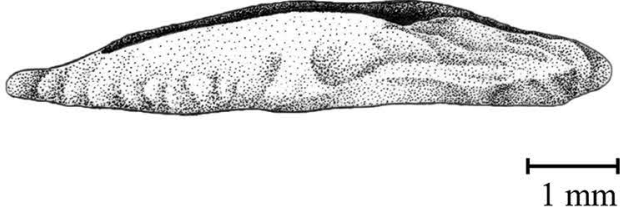

A1

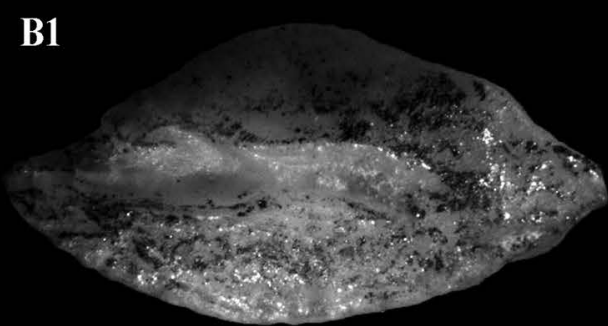

A2

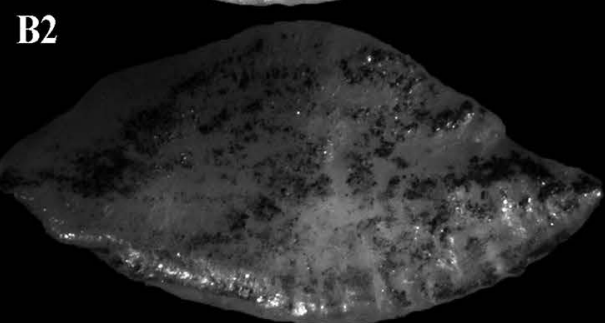

A3

\section{B3}

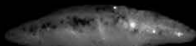

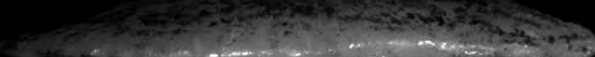

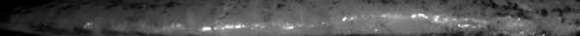

$$
1 \mathrm{~mm}
$$

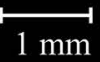

Plate 20. Illustrations (above) and photographs (below) of Scorpaena dispar right otoliths from fish with total lengths: A. $38 \mathrm{~mm}$; B. $129 \mathrm{~mm}$. The medial face is shown in A1, B1; the lateral face in A2, B2; and the ventral profile in A3, B3 (Illustration: Vanessa Sugihara; Photos: Cesar Santificetur). 
A1

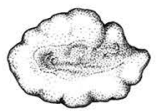

A2

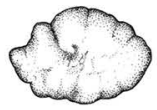

A3

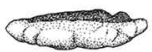

$\stackrel{\mathrm{mm}}{\longmapsto}$

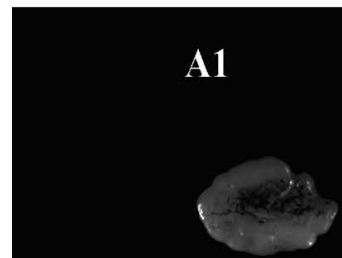

A2

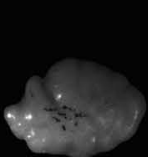

A3

(…

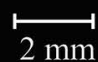

B1

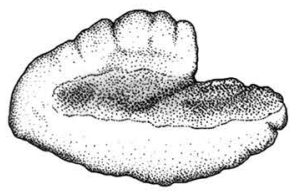

B2

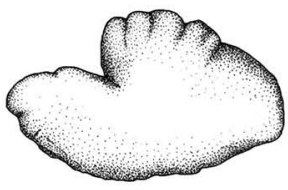

B3

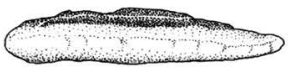

$\stackrel{\longmapsto}{2 \mathrm{~mm}}$

C3

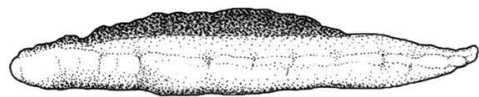

$2 \mathrm{~mm}$

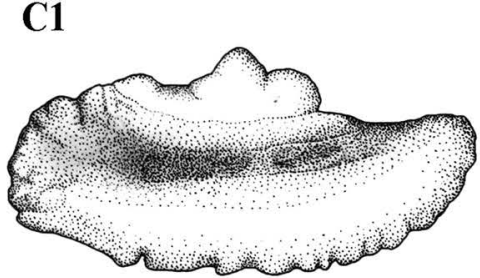

$\mathrm{C} 2$

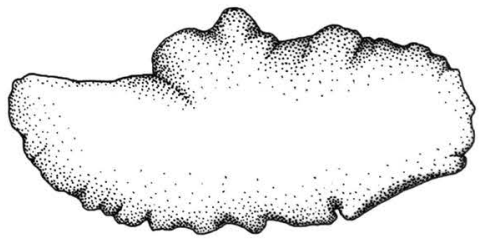

C1

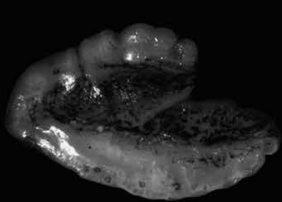

B2

$\mathrm{C} 2$

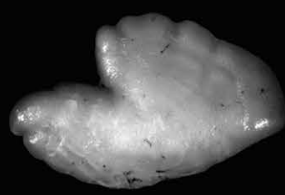

B3

C3

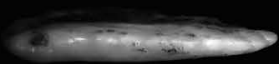

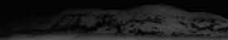

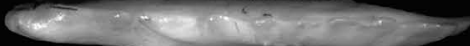

\section{$2 \mathrm{~mm}$}

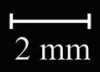

Plate 21. Illustrations (above) and photographs (below) of Setarches guentheri otoliths from fish with total lengths: A. 43 mm; B. 148 mm; C. 254 $\mathrm{mm}$. The medial face is shown in A1, B1, C1; the lateral face in A2, B2, C2; and the ventral profile in A3, B3, C3 (Illustration: Laura Montserrat; Photos: Cesar Santificetur). 
A1

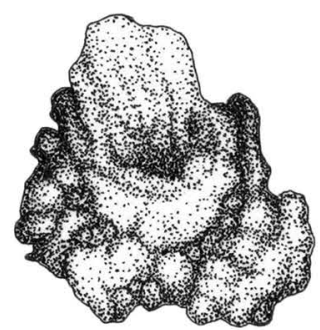

A2

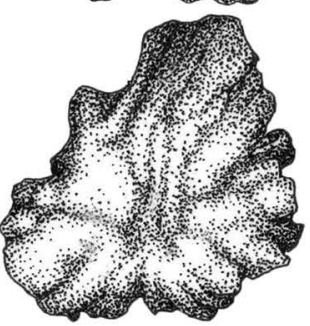

A3

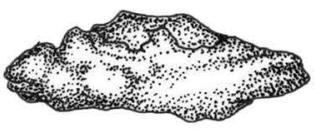

$\stackrel{\longmapsto}{\longmapsto m}$

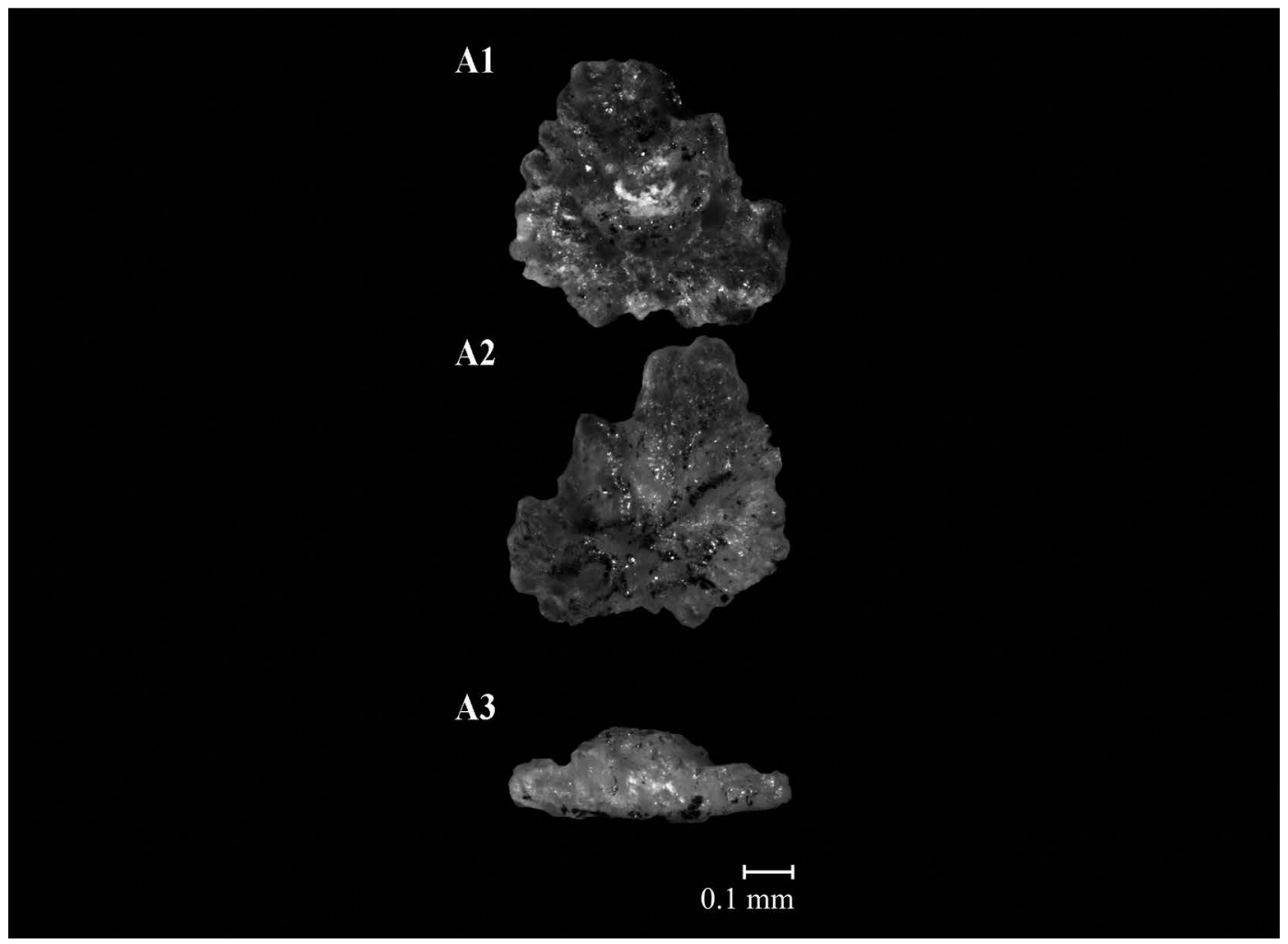

Plate 22. Illustrations (above) and photographs (below) of Chilomycterus spinosus otolith from fish with total length: A. $52 \mathrm{~mm}$. The medial face is shown in A1; the lateral face in A2; and the ventral profile in A3 (Illustration: Alexandre Arackawa; Photos: Cesar Santificetur). 
A1

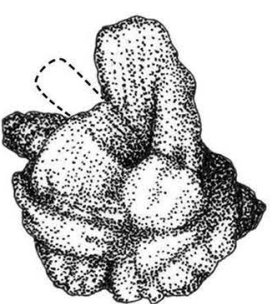

A2

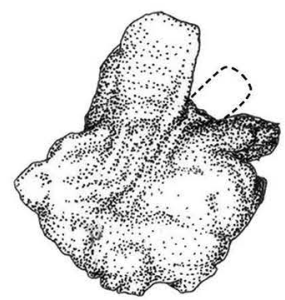

A3

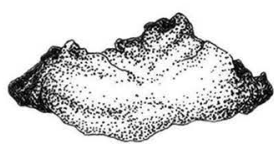

$0.2 \stackrel{\longmapsto \mathrm{mm}}{\longmapsto}$

A1

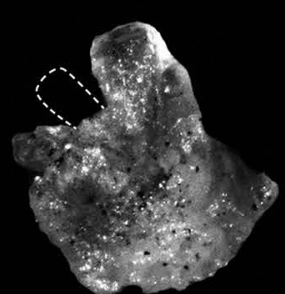

A2

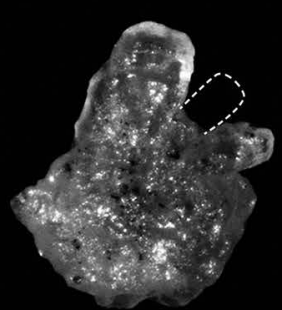

A3

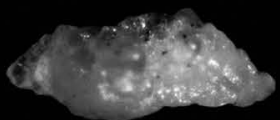

\section{$0.2 \stackrel{\mathrm{mm}}{\longmapsto}$}

Plate 23. Illustrations (above) and photographs (below) of Diodon holocanthus otolith from fish with total length: A. $111 \mathrm{~mm}$. The medial face is shown in A1; the lateral face in A2; and the ventral profile in A3 (Illustration: Alexandre Arackawa; Photos: Cesar Santificetur). 
A1

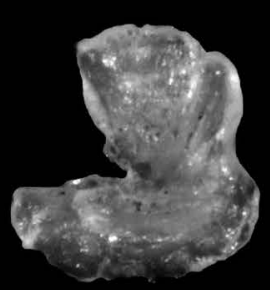

A2

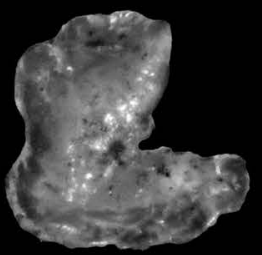

A3

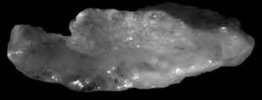

$0.5 \mathrm{~mm}$

A1

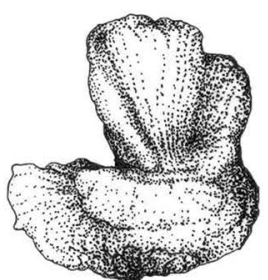

A2

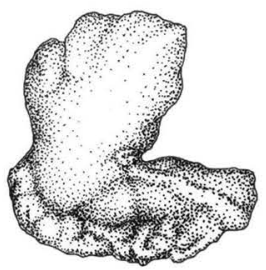

A3

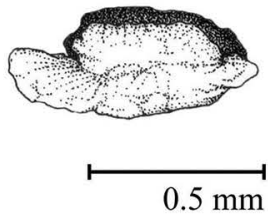

Plate 24. Illustrations (above) and photographs (below) of Lagocephalus laevigatus right otolith from fish with total length: A. 144 mm. The medial face is shown in A1; the lateral face in A2; and the ventral profile in A3 (Illustration: Alexandre Arackawa; Photos: Cesar Santificetur). 
A1

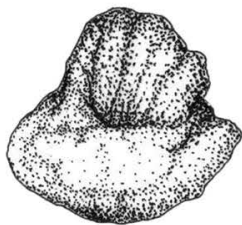

A2

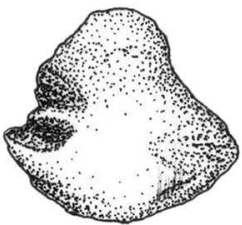

A3

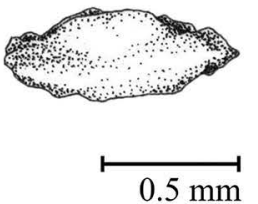

B1

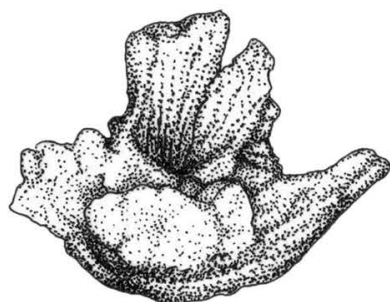

B2

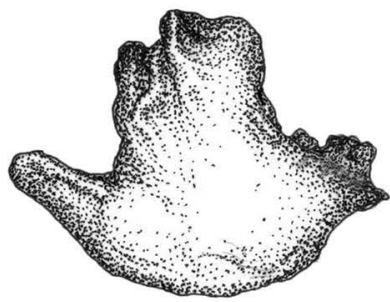

B3

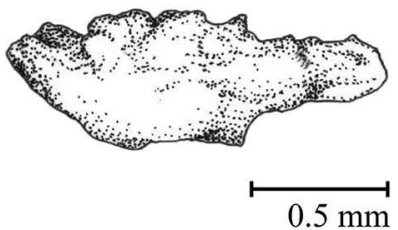

A1

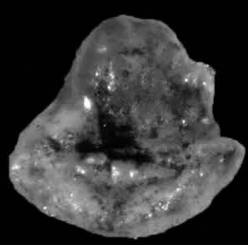

A2

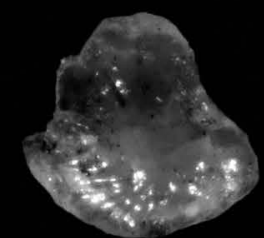

A3

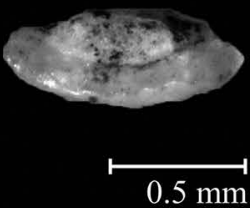

B1

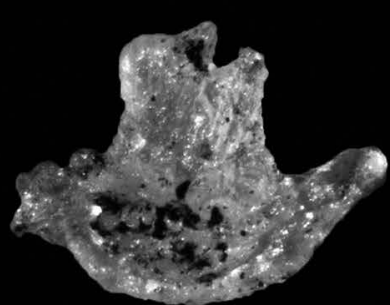

B2

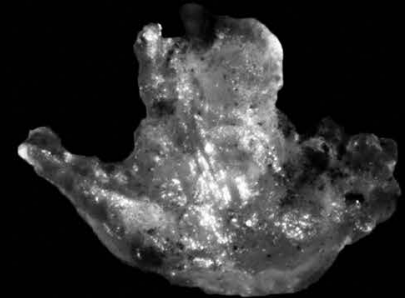

B3

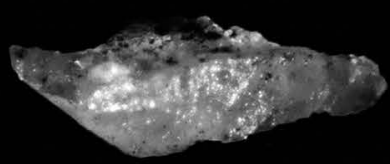

Plate 25. Illustrations (above) and photographs (below) of Sphoeroides greeleyi otoliths from fish with total lengths: A. $78 \mathrm{~mm}$; B. $145 \mathrm{~mm}$. The medial face is shown in A1, B1; the lateral face in A2, B2; and the ventral profile in A3, B3 (Illustration: Alexandre Arackawa; Photos: Cesar Santificetur). 
A1

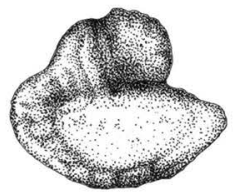

A2

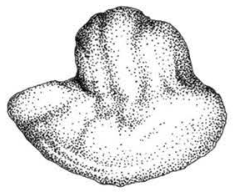

A3

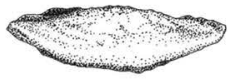

$0.2 \stackrel{\mathrm{mm}}{\longmapsto}$
B1

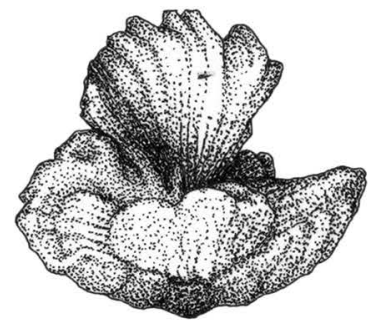

B2

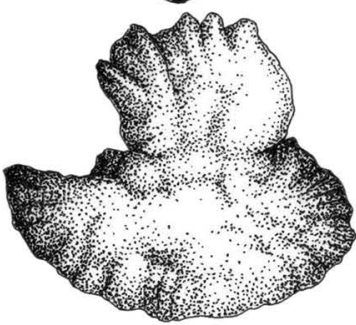

B3

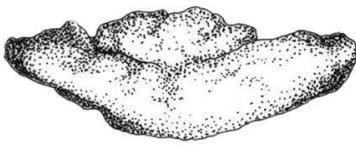

$0.2 \stackrel{\mathrm{mm}}{\longmapsto}$
A1

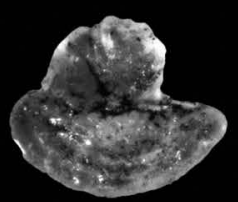

A2

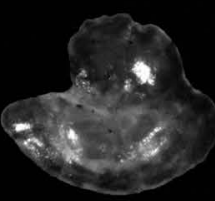

A3

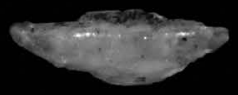

$0.2 \stackrel{\longmapsto}{\mathrm{mm}}$
B1

B2
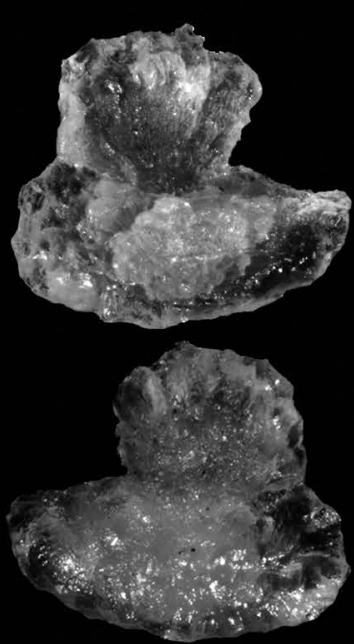

B3

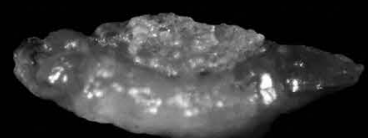

$2 \stackrel{1 \mathrm{~mm}}{\longmapsto}$

Plate 26. Illustrations (above) and photographs (below) of Sphoeroides testudineus otoliths from fish with total lengths: A. $94 \mathrm{~mm}$; B. $212 \mathrm{~mm}$. The medial face is shown in A1, B1; the lateral face in A2, B2; and the ventral profile in A3, B3 (Illustration: Alexandre Arackawa; Photos: Cesar Santificetur). 
A1

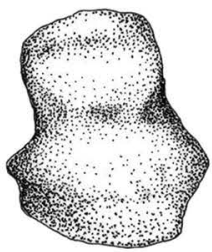

A2

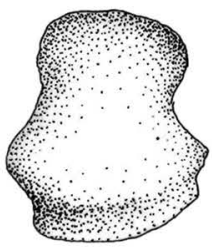

A3

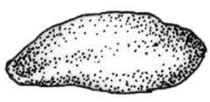

$0.5 \mathrm{~mm}$
B1

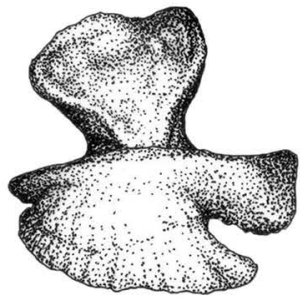

B2

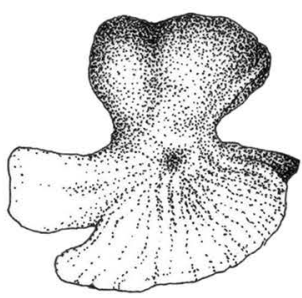

B3

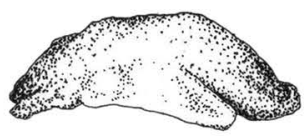

$0.5 \mathrm{~mm}$
A1

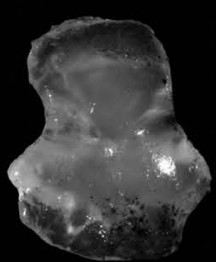

A2

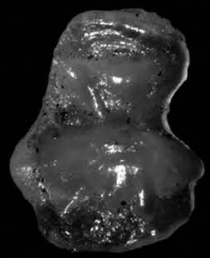

A3

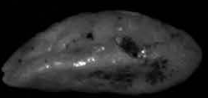

$0.5 \mathrm{~mm}$
B1

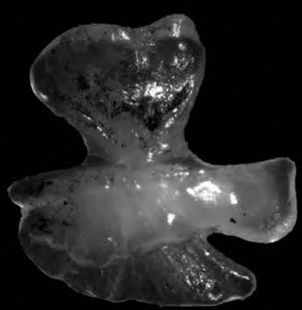

B2

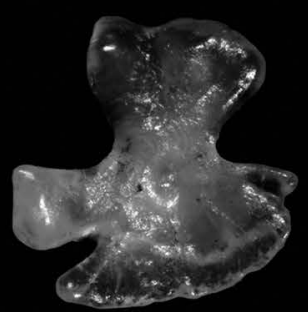

B3

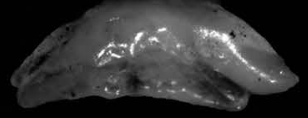

$0.5 \mathrm{~mm}$

Plate 27. Illustrations (above) and photographs (below) of Stephanolepis hispidus otoliths from fish with total lengths: A. 37 mm; B. 195 mm. The medial face is shown in A1, B1; the lateral face in A2, B2; and the ventral profile in A3, B3 (Illustration: Alexandre Arackawa; Photos: Cesar Santificetur). 